\title{
Impact of cosmic rays and solar energetic particles on the Earth's ionosphere and atmosphere
}

\author{
Peter I.Y. Velinov ${ }^{1}$, Simeon Asenovski ${ }^{1}$, Karel Kudela ${ }^{2}$, Jan Lastovicka ${ }^{3}$, Lachezar Mateev ${ }^{1}{ }^{*}$, Alexander Mishev ${ }^{4,5}$, \\ and Peter Tonev ${ }^{1}$ \\ 1 Institute for Space Research \& Technology, Academy of Sciences, 1113 Sofia, Bulgaria \\ *Corresponding author: e-mail: Inmateev@bas.bg \\ 2 Institute of Experimental Physics, SAS, Watsonova 47, 04001 Košice, Slovakia \\ 3 Institute of Atmospheric Physics, ASCR, Bocni II, 14131 Prague, Czech Republic \\ 4 Institute for Nuclear Research and Nuclear Energy, Academy of Sciences, 1784 Sofia, Bulgaria \\ 5 Sodankyla Geophysical Observatory, University of Oulu unit, Finland
}

Received 5 June 2012 / Accepted 4 March 2013

\begin{abstract}
A brief review of the study during COST Action ES0803 of effects due to cosmic rays (CR) and solar energetic particles (SEP) in the ionosphere and atmosphere is presented. Models CORIMIA (COsmic Ray Ionization Model for Ionosphere and Atmosphere) and application of CORSIKA (COsmic Ray SImulations for KAscade) code are considered. They are capable to compute the cosmic ray ionization profiles at a given location, time, solar and geomagnetic activity. Intercomparison of the models, as well as comparison with direct measurements of the atmospheric ionization, validates their applicability for the entire atmosphere and for the different levels of the solar activity. The effects of CR and SEP can be very strong locally in the polar cap regions, affecting the physical-chemical and electrical properties of the ionosphere and atmosphere. Contributions here were also made by the anomalous $\mathrm{CR}$, whose ionization is significant at high geomagnetic latitudes (above $65^{\circ}-70^{\circ}$ ). Several recent achievements and application of CR ionization models are briefly presented. This work is the output from the SG 1.1 of the COST ES0803 action (2008-2012) and the emphasis is given on the progress achieved by European scientists involved in this collaboration.
\end{abstract}

Key words. cosmic rays - solar energetic particles - ionization - ionosphere - atmosphere - solar activity - solar-terrestrial relationships

\section{Introduction}

The investigation of ionization processes in the ionosphere and atmosphere is important for better understanding of space weather mechanisms. The galactic cosmic rays (CR) influence the ionization and therefore the electrical parameters in the planetary atmospheres (Singh et al. 2011). They change also chemical processes - for example, ozone creation and depletion in the Earth's stratosphere (Brasseur \& Solomon 2005). In this way CR transfer the impact of solar activity into the atmosphere (Singh et al. 2010).

The lower part $(50-80 \mathrm{~km})$ of the ionospheric D-region is formed by the galactic CR, which create there an independent CR layer (Velinov 1968; Nestorov 1969; Velinov et al. 1974). However, the cosmic rays ionize the whole atmosphere up to $100 \mathrm{~km}$. Above this altitude the contribution of the electromagnetic $\mathrm{X}$ and $\mathrm{UV}$ radiations dominates. In such a way cosmic rays influence the ionization, chemical and electrical state in the region $5-100 \mathrm{~km}$. Near ground $(0-5 \mathrm{~km})$, there is an additional ionization source via natural radioactivity of the soil that may be important in some regions related to radon gas emission (Usoskin et al. 2011).

Three main components are important for the particle ionization: (1) high-energy galactic CR (GCR with energies $\sim 10^{9}-$ $10^{21} \mathrm{eV}$ ) that are always present in the Earth environment and are subject to 11-year solar modulation (Kudela 2009), (2) anomalous CR (ACR with lower energies $\sim 10^{6}-10^{8} \mathrm{eV}$ ) at high geomagnetic latitudes above $65^{\circ}-70^{\circ}$ and (3) sporadic solar energetic particles (SEP) of energy $\sim 10^{6}-10^{10} \mathrm{eV}$ ). Over the years solar energetic particles have been referred by a number of descriptive names such as solar cosmic rays, solar proton events and others (Miroshnichenko 2001, 2008; Dorman 2004; Mertens et al. 2012). The effect of the three components (GCR, ACR and SEP) is quantitatively studied in the present paper.

This year, 2012, marks the centenary anniversary of the discovery of cosmic rays by Victor Hess (Nobel Prize in Physics in 1936). Since then the scientific community is interested in the ionization effects of space radiation on the atmosphere and Earth's environment. The V2 rocket measurements in the middle of the 20th century by James Van Allen (1952) led to the first empirical profiles of the ionization effects until $100 \mathrm{~km}$ (Velinov et al. 1974). After that, the first quantitative models aiming to calculate the atmospheric ionization were developed (e.g., Velinov 1966, 1968; O’Brien 1971; Dorman \& Krupitskaya 1975). These models used a simplified approach based on an analytical approximation of the cosmic ray ionization losses. Therefore this approach was not so precise and accurate. Further improvement of these investigations was 
made by Velinov (1970, 1974), Dorman \& Kozin (1983), Velinov \& Mateev (1990), etc.

However at altitudes below $30 \mathrm{~km}$, the primary cosmic rays initiate a nucleonic-electromagnetic cascade in the atmosphere, with the main energy losses, resulting in ionization, dissociation and excitation of molecules (see, e.g., Dorman 2004). A principally new approach is the full Monte-Carlo CORSIKA (COsmic Ray SImulations for KAscade) simulation tool used for modelling the atmospheric nucleonic-electromagnetic cascade (Heck et al. 1998).

In the framework of the previous COST-724 action (20032007), three numerical CR ionization models have been developed (Usoskin et al. 2008, 2009):

1. The Sofia model includes an analytical approximation of the direct ionization by $\mathrm{CR}$ primaries above $30 \mathrm{~km}$ (Velinov et al. 2004, 2005a, 2005b, 2006, 2008; Buchvarova \& Velinov 2005) as well as the CORSIKA Monte-Carlo package extended by FLUKA package to simulate the low-energy nuclear interactions below 30 km (Velinov \& Mishev 2007, 2008a, 2008b; Mishev \& Velinov 2007, 2008).

2. The Oulu CRAC (Cosmic Ray Atmospheric Cascade) model is based on the CORSIKA/FLUKA Monte-Carlo simulations and explicitly accounting for direct ionization by primary CR particles (Usoskin et al. 2004, 2005; Usoskin \& Kovaltsov 2006; Usoskin et al. 2008, 2009).

3. The Bern model (ATMOCOSMICS/PLANETOCOSMICS code) is based on the GEANT-4 Monte-Carlo simulation package (Agostinelli et al. 2003). The main results are obtained by Desorgher et al. (2005), Scherer et al. (2007), Usoskin et al. (2008, 2009), etc.

Both codes (CORSIKA and GEANT-4) permit realistic study of the cascade evolution in the atmosphere, simulating the interactions and decays of various nuclei, hadrons, muons, electrons and photons. The result of the simulations is detailed information about the type, energy, momenta, location and arrival time of the produced secondary particles at given selected altitude above sea level (a.s.1.) (Usoskin et al. 2008, 2009).

Here we present some developments of these models (in particular, the Sofia model) during the COST ES0803 action (2008-2012), their validation and comparison with direct observations and other results. We also discuss effects caused by solar and galactic particles in the atmosphere.

\section{Model CORIMIA for CR ionization above $30 \mathrm{~km}$}

In contrast to the lower atmosphere, the ionization of the middle and upper atmosphere, where the cascade is not developed, allows a relatively simple analytical solution. This is related to the fact that the atmospheric depth at the altitude of $30 \mathrm{~km}$ is about $10 \mathrm{~g} / \mathrm{cm}^{2}$ (at $50 \mathrm{~km}$ is $1 \mathrm{~g} / \mathrm{cm}^{2}$ ), which is much less than the nuclear free path of protons and $\alpha$ particles $(\approx 70$ and $30 \mathrm{~g} / \mathrm{cm}^{2}$, respectively). Therefore, one can neglect nuclear interactions in the middle atmosphere above $30 \mathrm{~km}$ (upper stratosphere and ionosphere) and consider only ionization losses of the primary CR particles (Velinov et al. 1974; Usoskin et al. 2009). Moreover, for the altitude above $50 \mathrm{~km}$, one can further neglect changes of the energy of energetic particles, thus reducing the computation of $\mathrm{CR}$ ionization to an analytical thin target model (Velinov 1966, 1967a, 1968).

In the altitude range from $25-30$ to $50 \mathrm{~km}$, an intermediate target model needs to be used, that accounts also for the particle's deceleration due to ionization losses (e.g., Velinov 1967b; Velinov \& Mateev 1990). This model was applied for calculation of electron density and atmospheric electrical conductivities in the middle atmosphere for different particles: GCR, ACR and SEP. The intermediate target ionization model was further developed with account of the Chapman function values for the inclined penetrating particles in the spherical atmosphere (Velinov \& Mateev 2008a, 2008b; Velinov et al. 2008, 2009).

The program CORIMIA (COsmic Ray Ionization Model for Ionosphere and Atmosphere) is developed for calculation of the electron and ion production rate profiles due to cosmic rays using ionization losses (Bohr-Bethe-Bloch function) approximation in six characteristic energy intervals, including the charge decrease interval for electron capturing (Velinov et al. 2011a, 2011b, 2012).

Here some of results of the detailed model for calculation of $\mathrm{CR}$ ionization rates $q$ - the number of electron-ion pairs in $\mathrm{cm}^{-3}$ per second at given altitude $h(\mathrm{~km})$ in the ionosphere and middle atmosphere are presented. The mathematical expression of the fully operational program CORIMIA is the following (Velinov 1966):

$$
\begin{aligned}
q(h)= & \sum_{i} q_{i}(h) \\
= & \frac{1}{Q} \sum_{i} \int_{E_{i}}^{\infty} \int_{A=0}^{2 \pi} \int_{\theta=0}^{\frac{\pi}{2}+\Delta \theta} D_{i}(E)\left(\frac{\mathrm{d} E}{\mathrm{~d} h}\right)_{i} \\
& \times \sin \theta \mathrm{d} \theta \mathrm{d} A \mathrm{~d} E,
\end{aligned}
$$

where $D_{i}(E)$ is the $\mathrm{CR}$ differential spectrum $\left(\mathrm{cm}^{-2} \mathrm{~s}^{-1} \mathrm{st}^{-1} \mathrm{MeV}^{-1}\right),(\mathrm{d} E / \mathrm{d} h)$ are ionization losses (Sternheimer 1961) of particles of type $i, A$ is the azimuth angle, $\theta$ is the angle towards the vertical, $\Delta \theta$ takes into account that at a given height $h$ the particles can penetrate from the space angle $\left(0^{\circ}, \theta_{\max }=90^{\circ}+\Delta \theta\right)$, which is greater than the upper hemisphere angle $\left(0^{\circ}, 90^{\circ}\right)$ for flat model. $E_{i}$ are the energy cut-off which correspond to the geomagnetic cut-off rigidity $R_{c}$. The summation in the ionization integral (1) is made on the groups of nuclei $(i=1, \ldots, 6)$ : protons p, Helium ( $\alpha$ particles), Light L $(3 \leq Z \leq 5)$, Medium M $(6 \leq Z \leq 9)$, Heavy $\mathrm{H}(Z \geq 10)$ and Very Heavy $\mathrm{VH}(Z \geq$ 20) nuclei in the composition of cosmic rays. $Z$ is the charge of the nuclei, $Q=35 \mathrm{eV}$ is the energy which is necessary for formation of one electron-ion pair (Porter et al. 1976).

\subsection{Model description}

Five main characteristic energy intervals and one charge decrease interval for electron capturing in the approximation of ionization losses $\left(\mathrm{MeV} \mathrm{g} \mathrm{g}^{-1} \mathrm{~cm}^{2}\right.$ ) according to Bohr-BetheBloch formula using experimental data (Sternheimer 1961) are introduced (Velinov et al. 2008, 2009). The approximation for CR nuclei $(Z>1)$ is the following (Velinov et al. 2011b, 2012): 


$$
-\frac{1}{\rho} \frac{\mathrm{d} E}{\mathrm{~d} h}=\left\{\begin{array}{ccc}
2.57 \times 10^{3} E^{0.5} & \text { if } k T \leq E \leq 0.15 \mathrm{MeV} / \mathrm{n} & \text {, interval 1 } \\
1540 E^{0.23} & \text { if } 0.15 \leq E \leq E_{a}=0.15 Z^{2} \mathrm{MeV} / \mathrm{n} & \text {, interval 2 } \\
231 \times Z^{2} E^{-0.77} & \text { if } E_{a} \leq E \leq 200 \mathrm{MeV} / \mathrm{n} & \text {,interval 3 } \\
68 \times Z^{2} E^{-0.53} & \text { if } 200 \leq E \leq 850 \mathrm{MeV} / \mathrm{n} & \text {,interval 4 } \\
1.91 \times Z^{2} & \text { if } 850 \leq E \leq 5 \times 10^{3} \mathrm{MeV} / \mathrm{n} & \text {,interval } 5 \\
0.66 \times Z^{2} E^{0.123} & \text { if } 5 \times 10^{3} \leq E \leq 5 \times 10^{6} \mathrm{MeV} / \mathrm{n} & \text {, interval 6. }
\end{array}\right.
$$

Here interval 2 of the expression (2) is the charge decrease interval for $\mathrm{CR}$ nuclei $(Z>1)$. For protons $(Z=1)$ this interval is not necessary and it falls off. $E$ is expressed in $\mathrm{MeV} /$ nucl. In this way the accuracy of the obtained results is improved in comparison with the previous three- and four-interval approximations (Velinov \& Mateev 2008a, 2008b; Velinov et al. 2011a).

The model can be realized in submodels which evaluate the GCR, ACR and SEP contributions with account of the ionization in the middle atmosphere and lower ionosphere. Other structures in these submodels are the different characteristic energy interval contributions in the total ionization. This model can investigate the impact of random differential spectrum energy intervals on the ionization in the ionosphere and middle atmosphere. For this purpose satellite measurements of differential spectra are used.

In this paper we investigate particularly the GCR ionization for minimal, moderate and maximal solar activity, because observations cover this whole range of variability. That is caused by the cosmic ray modulation by the solar wind (Dorman 2004). We perform decomposition for different groups of GCR nuclei and for different characteristic energy intervals. The properties of the model towards the ionization losses function boundaries are realized and studied.

\subsection{CR spectra}

The differential spectrum of GCR is described by power law:

$$
D(E)=K E^{-\gamma}
$$

with the spectral coefficient $\gamma \approx 2.75$ for protons and slightly smaller in magnitude for nuclei (Ginzburg \& Syrovatskii 1964; Hillas 1972; Berezinsky et al. 1984). Particles with energy below $20 \mathrm{GeV}$ are subject to solar modulation. Here $D(E)$ deviates from the power law (3) and the spectrum has the following form (Velinov 1991; Velinov et al. 2001):

$$
D(E)=K(0.939+E)^{-\gamma}\left(1+\frac{\alpha}{E}\right)^{-\beta} .
$$

The constant 0.939 is the energy of rest of proton. $K, \alpha$ and $\beta$ are parameters of the spectrum which must be determined. These parameters for protons and for solar minimum have the following values: $K \approx 18.5, \alpha \approx 1.64$ and $\beta \approx 0.78$. They show the influence of solar wind modulation into the GCR spectrum. It is clear that by relativistic energies of the particles $E » 0.939 \mathrm{GeV}$ formula (4) becomes (3).

Buchvarova \& Velinov (2010) and Buchvarova et al. (2011) propose an empirical model for differential spectra $D(E)$ of cosmic rays during different phases of solar cycle. In this model parameters are related to the observed CR intensity. The empirical model proposed here approximates theoretical and experimental CR spectra during 11-year solar cycle. In the model data are used which cover three solar cycles: 20, 22 and 23.

We use the local interstellar spectrum (LIS) from Burger et al. (2000) with modification by Usoskin et al. (2005). Experimental data for protons and $\alpha$ particles for the ascending part of solar cycle 20 (1965-1969) are from Hillas (1972) and Simpson (1992). The measurements with LEAP87 (Seo et al. 1991), IMAX92 (Menn et al. 2000) and CAPRICE94 (Boezio et al. 1999) are related to solar cycle 22 (1986-1996). LEAP87 concerns the ascending phase and IMAX92 and CAPRICE94 - the descending phase of solar cycle 22, near solar minimum. AMS98 (Alcaraz et al. 2000a, 2000b) experimental spectrum is near the solar minimum of cycle 23 (1996-2008). The modelled differential spectra $D(E)$ are compared with BESS measurements (Shikaze et al. 2007) covering the solar cycle 23 (1996-2008). These experimental spectra are fitted to the proposed empirical model. The modulated CR differential spectra are compared with force-field approximation to the one-dimensional transport equation and with solutions of two-dimensional CR transport equation (Buchvarova \& Velinov 2010).

For experimental spectra, the calculation of the model parameters is performed by Levenberg-Marquardt algorithm (Press et al. 1991), applied to the special case of least squares. The proposed model gives practical possibility for construction of CR spectra on the basis of experimental data from measurements. From solar minimum to solar maximum the value of $\alpha$ increases and $\beta$ remains almost constant (Buchvarova \& Velinov 2010). In general the used by us GCR spectra are practically equal to the spectra of Usoskin et al. (2005) and Usoskin \& Kovaltsov (2006).

\subsection{Results for GCR ionization}

Model CORIMIA is capable to compute the cosmic ray ionization profiles at a given location, time, solar and geomagnetic activity. First we will show the calculations in the cusp region $\left(R_{c}=0 \mathrm{GV}\right)$ at different altitudes $h(30-120 \mathrm{~km})$. In fact, these are the maximum values of ionization in the atmosphere of the Earth. The results for ionization rate profiles for the different groups of GCR nuclei are presented in Figure 1. The total ionization rate (Fig. 1B) is composed by the ionization rates from main groups of the GCR nuclei: protons, Helium ( $\alpha$ particles), Light and Medium (Fig. 1A), Heavy and Very Heavy (Fig. 1B).

The computational results are obtained with the well-known Wolfram Mathematica computer algebra system, version 7.0 (Wolfram, Mathematica 2008). The input data are involved in 

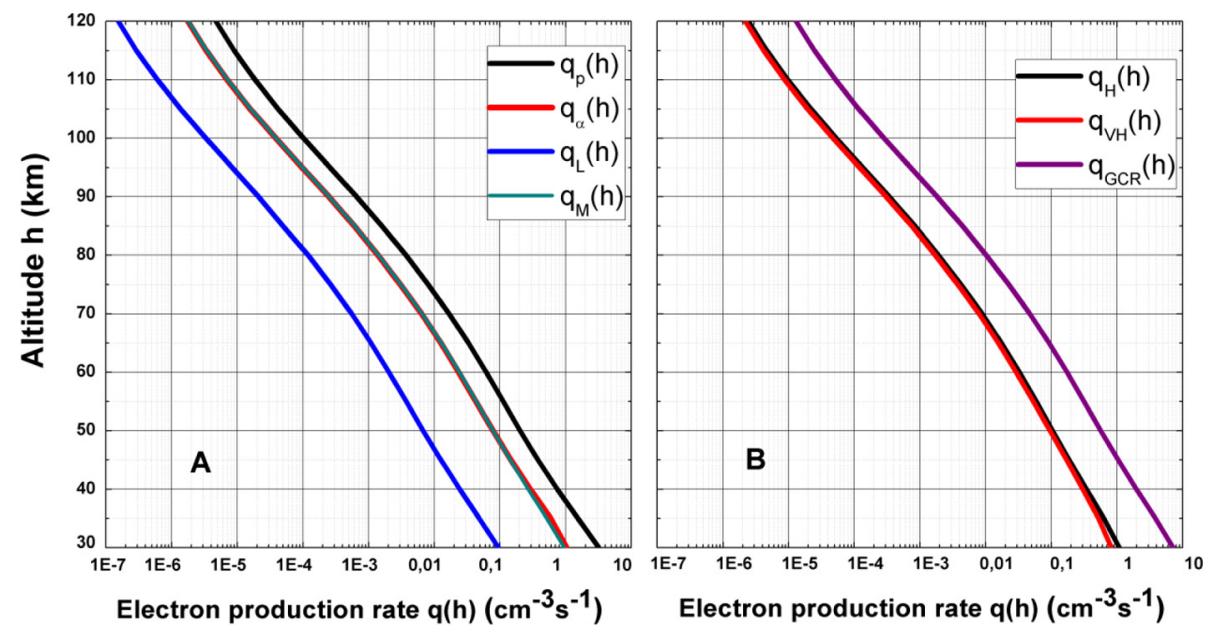

Fig.1. Electron production rate $q(h)$ profiles due to GCR in cusp region for $\mathbf{A}: \mathrm{p}, \boldsymbol{\alpha}, \mathrm{L}$ and $\mathrm{M}$ groups of nuclei, and $\mathbf{B}$ : $\mathrm{H}$ and $\mathrm{VH}$ groups of nuclei and the total GCR ionization during minimal solar activity.

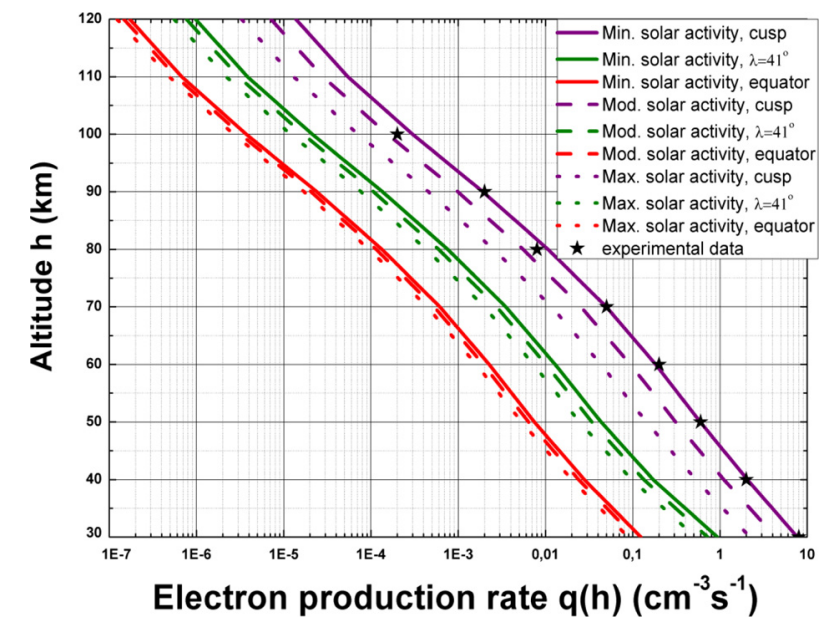

Fig. 2. Electron production rate $q(h)$ profiles due to GCR for cusp, middle latitudes $\left(\lambda=41^{\circ}\right)$ and equator at minimal, moderate and maximal solar activity. Experimental data $\left(^{*}\right)$ are taken from Brasseur \& Solomon (2005).

special input window. The output data are displayed in the corresponding output window. They show the inner structure of the model. The ionization profiles which are shown in Figures 1A and 1B show the maximal ionization in the Earth's ionosphere and atmosphere.

The calculations give a decrease of the ionization rates with the latitude (Fig. 2) because of increase of geomagnetic cut-off rigidity from geomagnetic poles $\left(R_{c}=0 \mathrm{GV}\right)$ to the geomagnetic equator $\left(R_{c} \approx 15 \mathrm{GV}\right)$. In Figure 2 are presented results for electron production rate $q(h)$ profiles for cusp (geomagnetic latitude $\left.\lambda=90^{\circ}\right)$, middle latitudes $\left(\lambda=41^{\circ}\right)$ and equator $\left(\lambda=0^{\circ}\right)$ at minimal, moderate and maximal solar activity. Experimental data $\left(^{*}\right)$ from rocket measurements (40$100 \mathrm{~km}$ ) are taken from Brasseur \& Solomon (2005). By reason of influence of solar wind modulation into the GCR, the atmospheric ionization decreases with growth of solar activity.

\subsection{Results for ACR ionization}

The ionization rates by the different ACR constituents are proportional to the magnitude of the corresponding differential

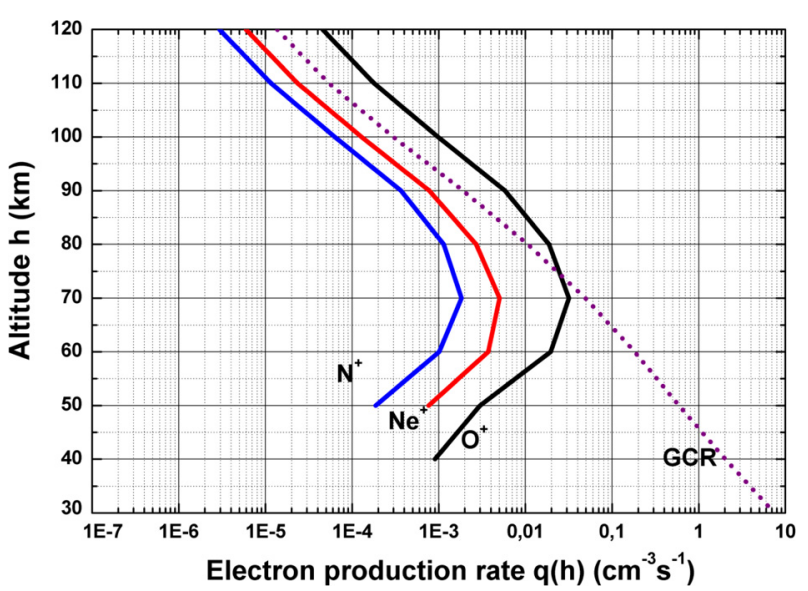

Fig. 3. Electron production rate $q(h)$ profiles calculated with CORIMIA for ACR main constituents: Nitrogen $(\mathrm{N})$, Oxygen $(\mathrm{O})$ and Neon (Ne) (Leske et al. 2011). GCR ionization $q(h)$ profile is at minimal solar activity (Fig. 1).

spectrum, neutral air density and the ionization rate energy interval values. Figure 3 presents the ionization rate profiles $q(h)$, calculated with CORIMIA, from the ACR main constituents: Nitrogen $(\mathrm{N})$, Oxygen $(\mathrm{O})$ and Neon $(\mathrm{Ne})$ (Leske et al. 2011). For comparison GCR ionization $q(h)$ profile at minimal solar activity (Fig. 1) is also given. We take recent experimental data for the ACR differential spectra from measurements of Advanced Composition Explorer (ACE) spacecraft at $1 \mathrm{AU}$ during the cycle 23/24 solar minimum (19 April 2009 to 20 November 2009; Leske et al. 2011).

The Cosmic Ray Isotope Spectrometer (CRIS) and Solar Isotope Spectrometer (SIS) onboard the ACE have been measuring GCR and ACR, respectively, since the launch of ACE in August 1997. These instruments provide a continuous, high-precision data set spanning, allowing detailed comparisons of cosmic ray modulation effects throughout more than an entire solar cycle (Leske et al. 2011).

For periods near the launch of ACE during the cycle 22/23 solar minimum in 1997/1998 and at the cycle 23/24 minimum in 2009, the constituents N, O and Ne have large ACR components; however $\mathrm{C}, \mathrm{Si}$ and $\mathrm{Fe}$ are without significant ACR contributions. 


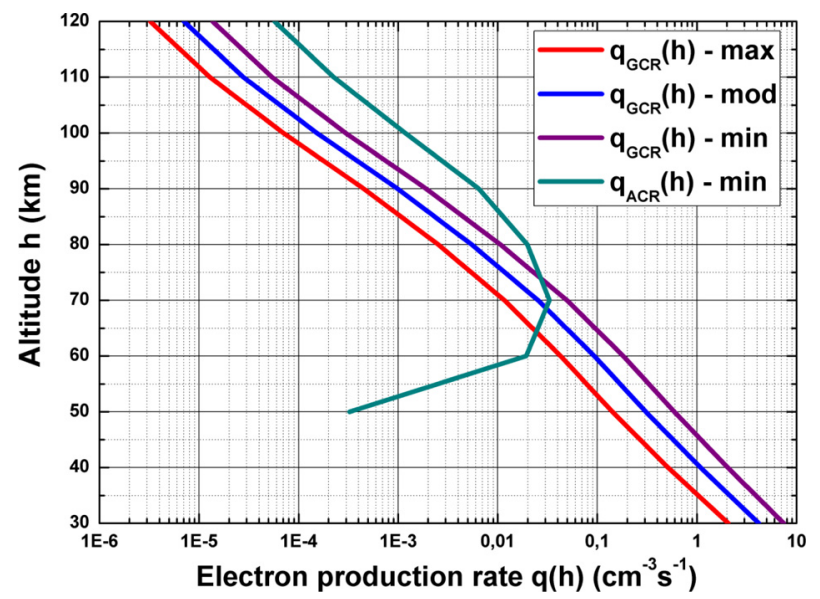

Fig. 4. Electron production rate $q(h)$ profiles in the cusp region calculated with CORIMIA for resultant $(\mathrm{N}+\mathrm{O}+\mathrm{Ne}$ from Fig. 3) ACR ionization and GCR ionization for minimal, moderate and maximal solar activity for cusp region from Figure 2 .

Figure 4 presents the resultant ionization profile $q(h)$ from the ACR main constituents: $\mathrm{O}+\mathrm{N}+\mathrm{Ne}$, shown in Figure 3. For comparison GCR ionization $q(h)$ profiles for minimal, moderate and maximal solar activity for cusp region (Fig. 2) are also given. From Figure 4 it can be seen, that the ACR contribution in the atmospheric ionization dominates over the GCR contribution at heights above $65 \mathrm{~km}$ at solar maximum and above $75 \mathrm{~km}$ at solar minimum.

The ACR spectrum is different for every ACR occurrence. In Figure 5 are shown results obtained with CORIMIA from another spacecraft data - from Voyager measurements in the beginning of its flight at $\mathrm{AU} \approx 1.3$. We can use these data according to McDonald et al. (2002). From their investigations it can be seen that the spectrum changes for this case in comparison with $\mathrm{AU}=1$ are small.

Here are presented electron production rate $q(h)$ profiles for ACR main constituents: Helium (He), Nitrogen (N), Oxygen (O) and Neon (Ne) (Cummings et al. 1984). We have used data from the CR subsystem on the Voyager spacecraft during solar minimum conditions near the end of 1977, when there minimum modulation of CR fluxes.

As Voyager has no data for protons for this period, we used other satellite data compiled by Simpson (1992) during similar conditions. The ACR spectra are obtained using the fitting procedure of Mathematica program system (Fit procedure which gives their analytical expressions) with the experimental data from spacecraft measurements (Wolfram Research 2008). We compute ACR spectra for $\mathrm{H}^{+}, \mathrm{He}^{+}, \mathrm{N}^{+}, \mathrm{O}^{+}$and $\mathrm{Ne}^{+}$with charge $Z=1$, i.e., singly ionized.

The ionization rate is calculated with CORIMIA code as a subroutine in the Mathematica program system (Wolfram Research 2008), which generates expressions for the corresponding input spectra for CORIMIA code which is started in it.

From Figure 5 is seen that the proton and Helium constituents have significant contributions in the total ACR ionization. For comparison GCR ionization $q(h)$ profile for minimal solar activity for cusp region (Fig. 1) is also shown. The ACR impact on the ionosphere and atmosphere is confined predominantly to the polar cap regions above geomagnetic latitude, approxi-

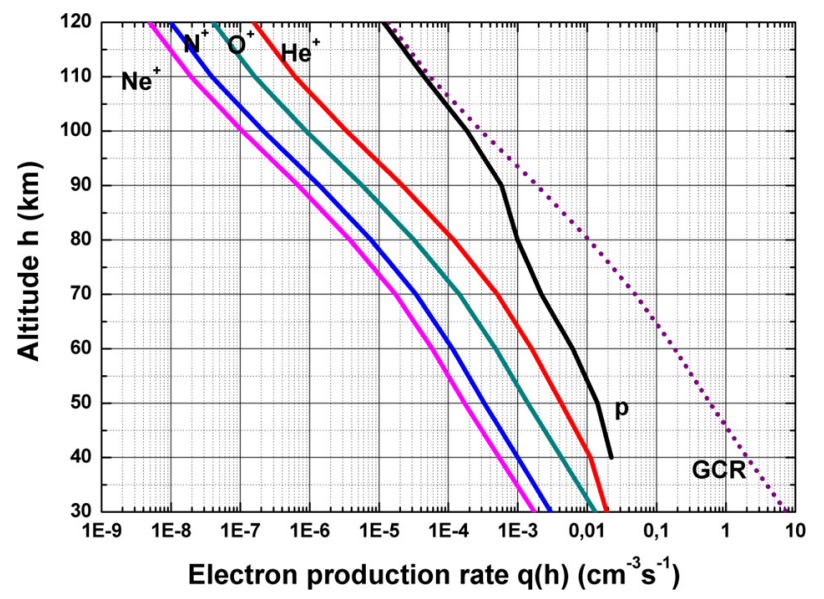

Fig. 5. Electron production rate $q(h)$ profiles calculated with CORIMIA for ACR main constituents: protons (p) (Simpson 1992); Helium (He), Nitrogen (N), Oxygen (O) and Neon (Ne) (Cummings et al. 1984). GCR ionization $q(h)$ profile is at minimal solar activity for cusp region (Fig. 1).

mately, $\lambda_{m}=65^{\circ}-70^{\circ}$. The ACR spectra are effective below $100 \mathrm{MeV}$. The GCR spectra have independent contribution above $100 \mathrm{MeV}$.

\subsection{Results for SEP ionization}

Occasionally the Sun emits relativistic energetic particles of sufficient energy and intensity to raise radiation levels on Earth's surface to the degree that they are readily detected by neutron monitors. Actually they cause Ground Level Enhancements (GLE) of cosmic rays. Since the energies of these solar relativistic particles (up to $\sim 10^{9}-10^{10} \mathrm{eV}$ ) are commensurate with the energies of galactic cosmic rays, sometimes they are called solar cosmic rays.

The present paper shows the results from CORIMIA program (Velinov et al. 2012) with application to the GLE 69 on 20 January 2005 and GLE 05 on 23 February 1956 (Reid 1961; Velinov et al. 1974). The corresponding differential spectra for GLE 69 are taken from the available GOES satellite data. We investigate the SEP effects in the polar cap region at geomagnetic latitudes $65^{\circ}-80^{\circ}$ during two of the most powerful solar events which have been observed since 28 February 1942. Then was registered GLE 01 and then began the study of SEP impacts. In this way the extreme influence of solar activity on ionization state of the ionosphere and middle atmosphere will be calculated.

Unlike the cases of GCR, SEP differential spectra vary essentially in time during the course of the investigated event. It is difficult to make a generalization of global solar influence on ionization, chemistry and electrical conductivities in atmosphere for the whole time period. That is why it is appropriate to consider more than one moment of SEP impact. For the case of GLE 69 we include two characteristic time points - at the beginning 8:00 UT and 23:00 UT. The corresponding differential spectrum in $\mathrm{cm}^{-2} \mathrm{~s}^{-1} \mathrm{MeV}^{-1}$ outside of the atmosphere (according GOES data) for the time point at 8:00 UT is:

$$
D(E)=1.55 \times 10^{6} E^{-2,32},
$$

and for the time point at 23:00UT the spectrum is:

$$
D(E)=10^{7} E^{-3.43} \text {. }
$$




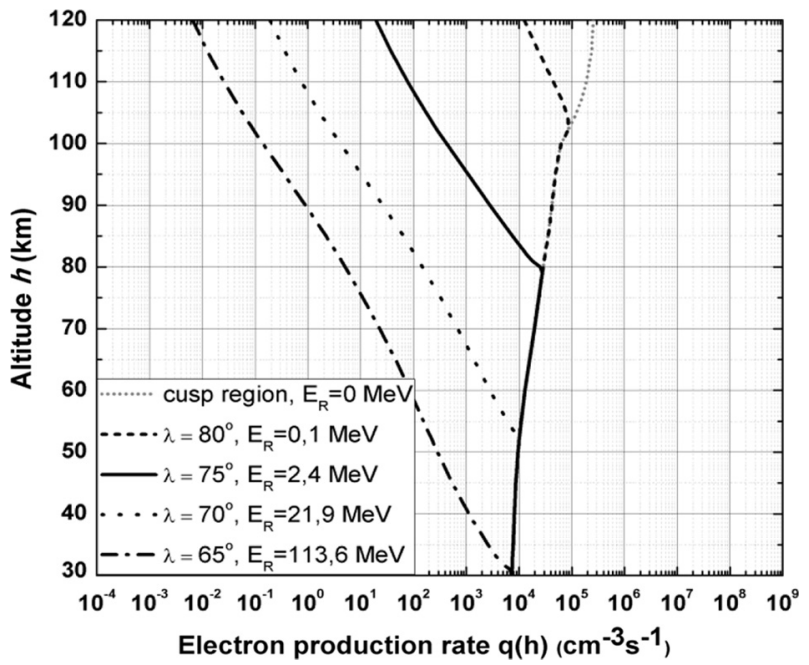

Fig. 6. Electron production rate $q(h)$ profiles due to SEP event during GLE 69 with spectrum measured on 20 January 2005 at 08:00 UT. $E_{\mathrm{R}}$ is energy corresponding to geomagnetic cut-off for protons.

The differential spectrum for GLE 05 (Reid 1961) is:

$$
D(E)=2.4 \times 10^{10} E^{-5} .
$$

The energy $E$ in Eqs. (5)-(7) is expressed in MeV/nucl. These spectra are obtained in the following way. Two data points are taken from the GOES data lists for protons in Internet for every spectrum. They belong to different energy intervals of measurement as given in these data lists. After that a system of equations is solved towards both unknown parameters of spectrum: the magnitude and exponent.

For the first time CORIMIA program is applied to the GLE and results show that it is suitable for the calculations of the ionization effects from solar particles. The model embedded in this program includes the full approximation (2) of the Bohr-BetheBloch formula (Velinov et al. 2011b, 2012; Dorman 2004) using six characteristic energy intervals for CR nuclei groups.

We investigate the case of solar proton penetration (charge $Z=1$ ) in the Earth's atmosphere. That means interval 2 is not taken into account. On the other hand, we find out that the last three high-energy intervals (above $200 \mathrm{MeV}$ ) do not have contributions to the ionization rate (GLE 69 at 23:00 UT and GLE 05) (NOAA Space Weather Prediction Center - GOES satellite). The last two intervals approximately (the energies above $2 \mathrm{GeV}$ ) for GLE 69 at 8:00 UT are also without contribution. The dependence of number of particles on the characteristic energy intervals influences the ionization rate profiles (3).

The SEP submodel for calculation of ionization rates caused by solar particles in the ionosphere and atmosphere with account of first three characteristic energy intervals is applied for the GLE 69 and GLE 05 events. In Figures 6, 7 and 8 the main results are presented as calculated with CORIMIA program. Figures 6 and 7 give the ionization rate $q(h)$ profiles caused by SEP during GLE 69 with spectra measured on 20 January 2005 at 8:00 UT and 23:00 UT. These profiles reflect the ionization state in the polar oval region for $\lambda_{\mathrm{m}}=65^{\circ}$, $70^{\circ}, 75^{\circ}, 80^{\circ}$ and the corresponding geomagnetic cut-offs there. The altitude range includes the height interval $30-120 \mathrm{~km}$. As it can be seen in Figures 6 and 7 the profile maxima altitudes grow with latitude.

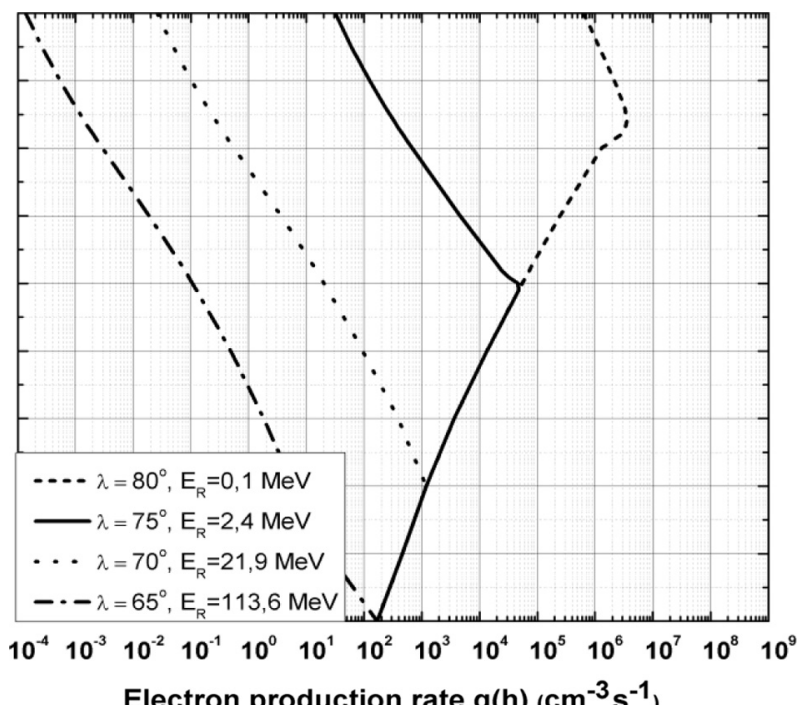

Electron production rate $q(h)\left(\mathrm{cm}^{-3} \mathrm{~s}^{-1}\right)$

Fig. 7. Electron production rate $q(h)$ due to SEP event during GLE 69 with spectrum measured on 20 January 2005 at 23:00 UT. $E_{\mathrm{R}}$ is energy corresponding to geomagnetic cut-off for protons.

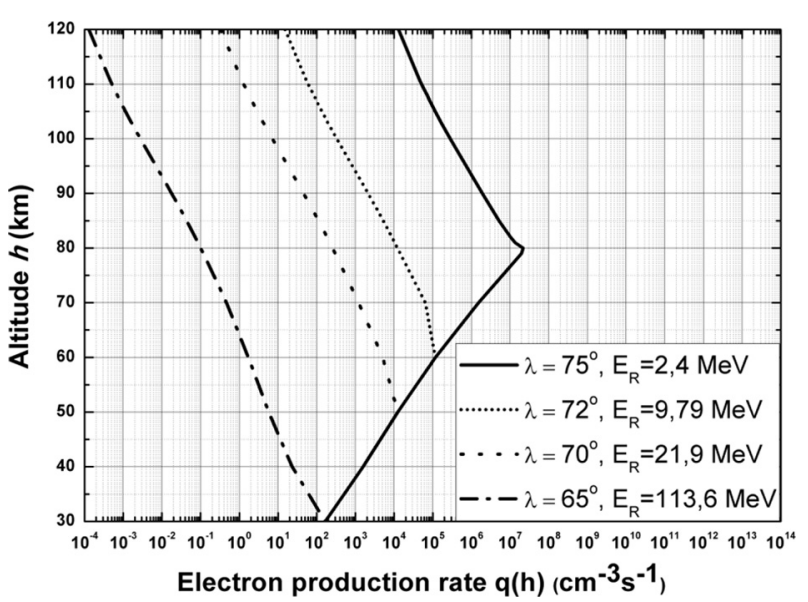

Fig. 8. Electron production rate $q(h)$ profiles due to SEP event during GLE $05 . E_{\mathrm{R}}$ is energy corresponding to geomagnetic cut-off for protons.

Figure 8 presents the results of electron production rate calculation of the GLE 05 on 23 February 1956. This is the most powerful solar proton event which has ever been observed in the history of space research. Similar to other two spectra from 20 January 2005 in this case there is an increase of maximum altitude with increasing latitude.

The CORIMIA program is able to calculate the ionization rates stably and accurately for the effects of any SEP impact on the lower ionosphere and middle atmosphere. Its structure is user friendly developed with detailed description of input and output data in corresponding windows. In the future we will develop and improve the CORIMIA program as directly applicable routine for the goals of the space weather investigation.

\section{Recent Monte-Carlo models for CR ionization}

The full Monte-Carlo simulations of CR ionization are related to explanation and modeling of different processes in the 
atmosphere (Bazilevskaya et al. 2008) as well as their complex experimental study (Mishev 2010). At present with the development of numerical methods and evolution of the knowledge of high-energy interactions and nuclear processes, an essential progress in models for cosmic ray ionization processes in the Earth atmosphere is carried out (Desorgher et al. 2005; Usoskin \& Kovaltsov 2006; Mishev \& Velinov 2007; Velinov et al. $2009,2011 b)$. These precise models are based on full MonteCarlo simulation of the atmospheric cascade. The models agree with $10-20 \%$, the difference is mainly due to the various hadron generators and atmospheric models (Usoskin et al. 2009).

The Monte-Carlo codes permit to follow the longitudinal cascade evolution in the atmosphere and obtain the energy deposit by different shower components and particles from ground till the upper atmosphere. These full target models apply the formalism of ionization yield function $Y(h, E)$ (the number of ion pairs produced at altitude $h$ by one primary cosmic ray nucleus with kinetic energy $E$ on the top of the atmosphere). The estimation of atmospheric ion rate production is based on equation (Usoskin et al. 2009):

$$
q\left(h, \lambda_{m}\right)=\int_{E_{0}}^{\infty} D\left(E, \lambda_{m}\right) Y(h, E) \rho(h) \mathrm{d} E,
$$

where $D(E)$ is the differential primary cosmic ray spectrum at a given geomagnetic latitude for a given component of primary cosmic ray, $Y$ is the yield function and $\rho(h)$ is the atmospheric density $\left(\mathrm{g} \mathrm{cm}^{-3}\right)$.

\subsection{An extension of CRAC ionization model to the upper atmosphere}

Ionization of the upper atmosphere is important for the atmospheric chemistry and dynamics (Krivolutsky et al. 2005; Lastovicka \& Krizan 2005). The ionization in the upper atmosphere is usually computed using an analytical approximation (Vitt \& Jackman 1996) and is typically focused upon the energy range of primary cosmic rays below $500 \mathrm{MeV}$ (Wissing \& Kallenrode 2009). These models are applicable on sporadic and highly temporary variable ionization effect of solar energetic particles and are less suitable to study galactic cosmic rays.

In this connection the Oulu model was extended to the upper atmosphere (Usoskin et al. 2010). The extension of the model is based on a well-known full Monte-Carlo simulation of the atmospheric cascade and an additional thin target analytical approach. In the model the elastic scattering is neglected and it is assumed that the particle moves straight, but loses its energy due to ionization of the ambient air or to nuclear inelastic processes. The full details as well as comparisons are given by Usoskin et al. (2010). A detailed comparison for level of $100 \mathrm{~g} \mathrm{~cm}^{-2}$ of the atmosphere due to galactic cosmic ray protons computed for solar maximum and minimum is carried out. It is demonstrated that considering only low-energy protons $(<500 \mathrm{MeV})$ the ionization effect is underestimated by a factor of 2 for the solar minimum and a factor of 5 for the solar maximum conditions, even in the uppermost part of the atmosphere. The difference is larger for the solar maximum because of the harder energy spectrum of galactic cosmic rays.

When a simplified analytical model is used instead of a full solution, the underestimation of the ionization rate at $0.1 \mathrm{~g} \mathrm{~cm}^{-2}$ is $10 \%$, at $1 \mathrm{~g} \mathrm{~cm}^{-2}$ is $20-25 \%$ and at $10 \mathrm{~g} \mathrm{~cm}^{-2}$ - by a factor of 2 . At the atmospheric depth greater than a few tens of $\mathrm{g} \mathrm{cm}^{-2}$ (altitudes below about $20 \mathrm{~km}$ ) the analytical model is not correct. Another significant result from this study is related to modeling of ionization effect due to solar energetic particles. As was demonstrated the analytical model considering only protons with energy below $500 \mathrm{MeV}$ well corresponds with full model computation at about $40 \mathrm{~km}$ altitude. Below the analytical model progressively underestimates the ionization effect.

\subsection{Effect of model assumptions on computations of CR ionization in atmosphere}

As it was recently shown, various Monte-Carlo models agree within 10-20\% (Usoskin et al. 2009). In this connection their investigation is crucial for further modeling of various processes. The largest uncertainties in numerical simulation of atmospheric cascades are due to the assumed models for hadron interactions. Therefore the influence of low-energy hadron interaction models in CORSIKA code on the energy deposition, respectively, ionization is very important. In the past mostly GHEISHA (Fesefeldt 1985) routines have been used for simulations of atmospheric cascades. However it is known that GEANT-GHEISHA suffers from deficiencies in handling the reaction kinematics properly (Ferrari \& Sala 1996). As example GHEISHA and FLUKA (Battistoni et al. 2007) predict different momentum distributions of secondary $\pi \pm$ mesons.

The largest differences are observed between the energy spectra amount (up to $\approx 15 \%$ ) at $E \mu \approx 0.8 \mathrm{GeV}$ and they are clearly correlated with the differences in the predicted distributions of $\pi$-mesons at $x_{\text {lab }} \approx 0.15$. Another difference of $\approx 10 \%$ is observed at $E \mu \approx 10 \mathrm{GeV}$ related to the distribution of charged pions in $\pi \pm{ }^{14} \mathrm{~N}$ collisions at $x_{\text {lab }} \approx 0.6$. While the electron densities of simulated cascades show no significant dependence on the low-energy model used, its influence on the hadronic and muonic component is obvious.

A comparison between GHEISHA 2002 (Fesefeldt 1985) and FLUKA 2006b (Battistoni et al. 2007) was carried out (Mishev \& Velinov 2007). It was demonstrated by Mishev \& Velinov (2010, Fig. 2) that the largest differences are observed for the contribution of hadron and muon component specifically for $10 \mathrm{GeV} /$ nucleon and $1 \mathrm{TeV} /$ nucleon energy of the primary proton. However, the difference in total ionization is not so crucial and both models are applicable, specifically below the Pfotzer maximum (Fig. 9). This is mainly due to the cascade process multiplicity (Figs. 9C and 9D), the hard steep spectrum of GCR and the dominant contribution of various secondary components as a function of altitude and rigidity cut-off (Figs. 9A-9D).

Similar comparison is performed for various atmospheric profiles (Mishev \& Velinov 2008). The comparison is carried out for summer, winter and US standard atmospheric profile (Fig. 10). The main reason to compare the cosmic ray induced ionization is the observed seasonal variation of the atmospheric profiles and the observed differences in altitudes of shower maximum (Keilhauer et al. 2004, 2006). The obtained ionization yield functions $Y$ are the same below $200 \mathrm{~g} \mathrm{~cm}^{-2}$ for winter, summer and US standard atmospheric profiles in the case of $1 \mathrm{GeV}$ incoming protons (Fig. 10A). In the region of Pfotzer maximum the ionization rate tends to slightly increase for winter profile (Figs. 10B and 10C). Generally the ionization rate for US standard atmospheric profile lies between the rates for winter and summer profiles. The obtained results permit a realistic modeling of a given event. 

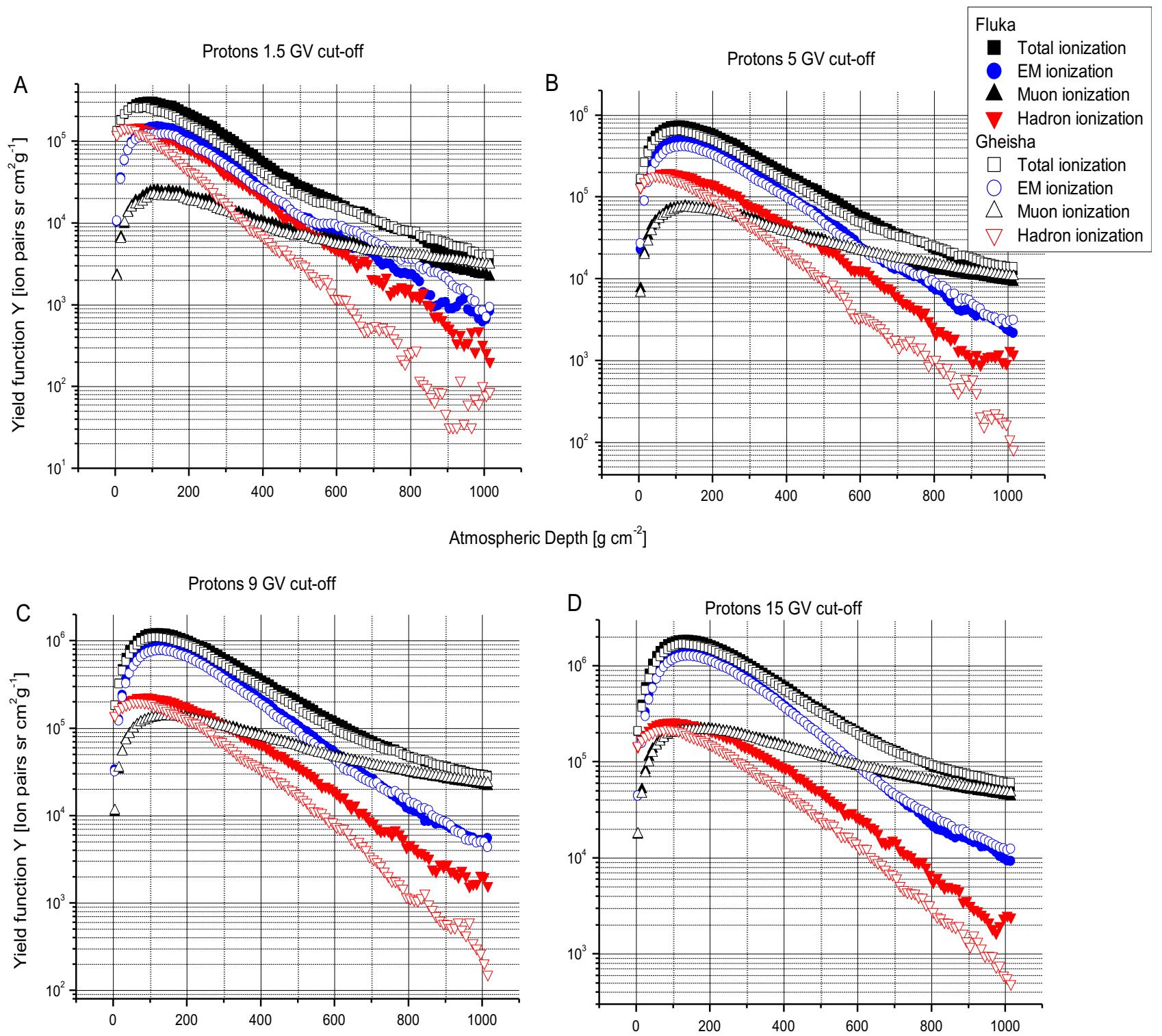

Atmospheric Depth $\left[\mathrm{g} \mathrm{cm}^{-2}\right]$

Fig. 9. Ionization yield function $Y$ for primary proton induced atmospheric cascades with different cut-offs $(1.5,5,9$ and $15 \mathrm{GV})$ simulated with FLUKA and GHEISHA hadron generators.

\subsection{Normalization of ionization yield function}

As was recently shown the specific ionization yield functions are different for various primary nuclei, particularly in the upper atmosphere (Mishev \& Velinov 2007, 2009; Velinov \& Mishev 2007). This is due to specifics of atmospheric cascade development and direct ionization of the initiated particles. For correct estimation the contribution to the ionization of each nucleon of the cascade, it is necessary to simulate large variety of primaries. An issue is the normalization of ionization yield function proposed by Usoskin \& Kovaltsov (2006). The normalization consists of presentation of ionization yield function $Y$ as ion pairs per nucleon (Mishev \& Velinov 2010, 2011a, 2011b, 2012).

The normalized ionization yield functions are still different in the upper atmosphere. They are the same below $24 \mathrm{~km}$ above sea level for $1 \mathrm{GeV} /$ nucleon primary nuclei (Fig. 11A), below about $23 \mathrm{~km}$ a.s.l. for $10 \mathrm{GeV} /$ nucleon primary nuclei (Fig. 11B) and $22 \mathrm{~km}$ a.s.l. for $100 \mathrm{GeV} /$ nucleon primary nuclei (Fig. 11C). Therefore in the sense of produced ionization below these levels, heavier nuclei can be considered as identical to the corresponding number of $\alpha$ particles, i.e. an oxygen nucleus can be substituted by $4 \alpha$-particles, an iron nucleus can be substituted by $14 \alpha$-particles, respectively (Usoskin \& Kovaltsov 2006; Mishev \& Velinov 2011a, 2011b). Hence for quantitative studies, especially at low altitudes it is possible to apply this convention, which simplifies considerably the simulation. However in the region of the upper atmosphere, the difference is essential and the contribution of various nuclei to the atmospheric ionization should be considered individually (Mishev \& Velinov 2011a, 2011b).

\subsection{Ionization effect in the atmosphere during GLE}

In addition to continuous ionization in the Earth's atmosphere caused by galactic cosmic rays a sporadic ionization occurs during solar energetic particle events, potentially affecting the 
P.I.Y. Velinov et al.: Impact of CR and SEP on the Earth's ionosphere and atmosphere

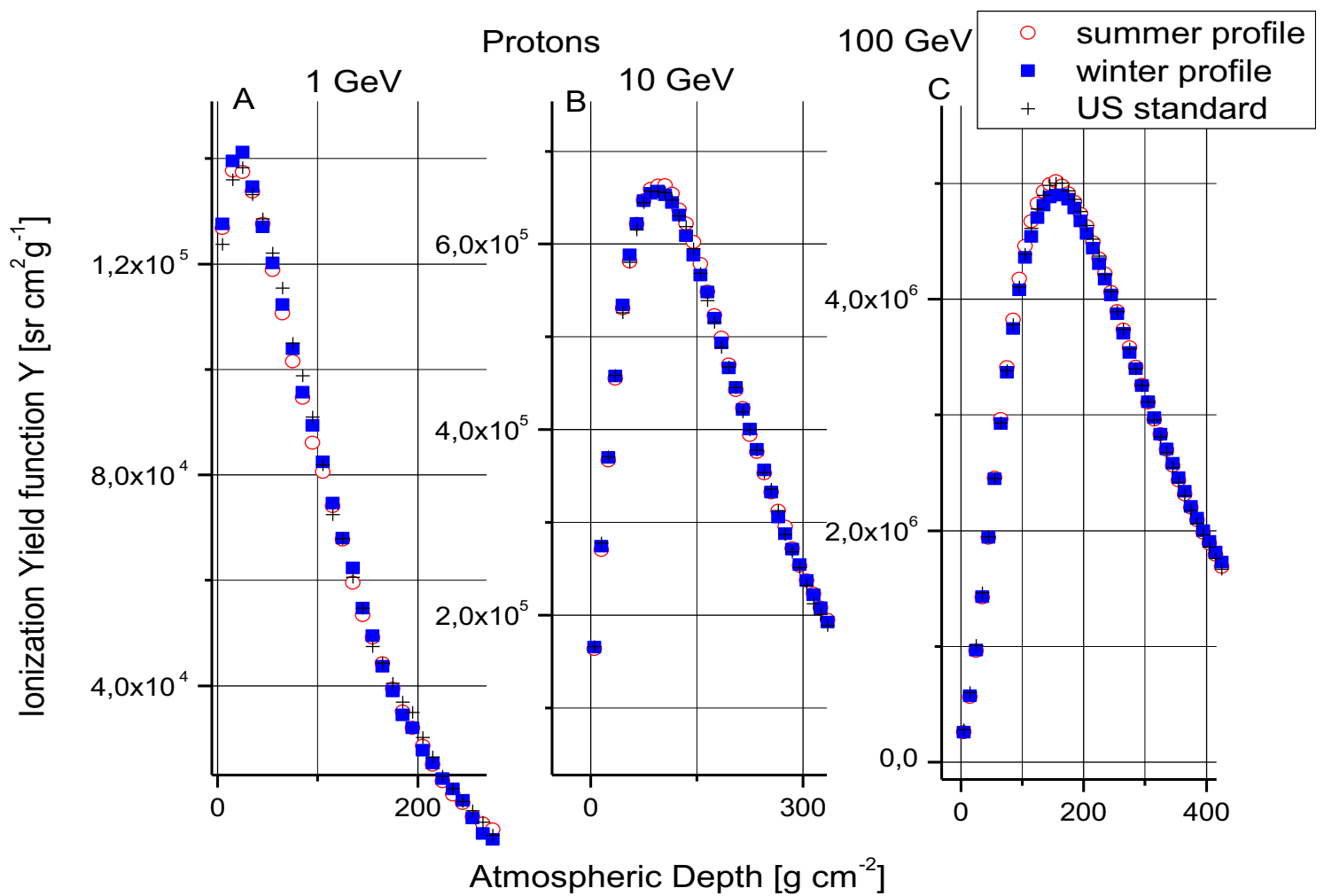

Fig. 10. Ionization yield function $Y$ computed for winter, summer and US standard atmospheric profile as a function of the energy (1, 10 and $100 \mathrm{GeV}$ ) of primary proton nuclei.
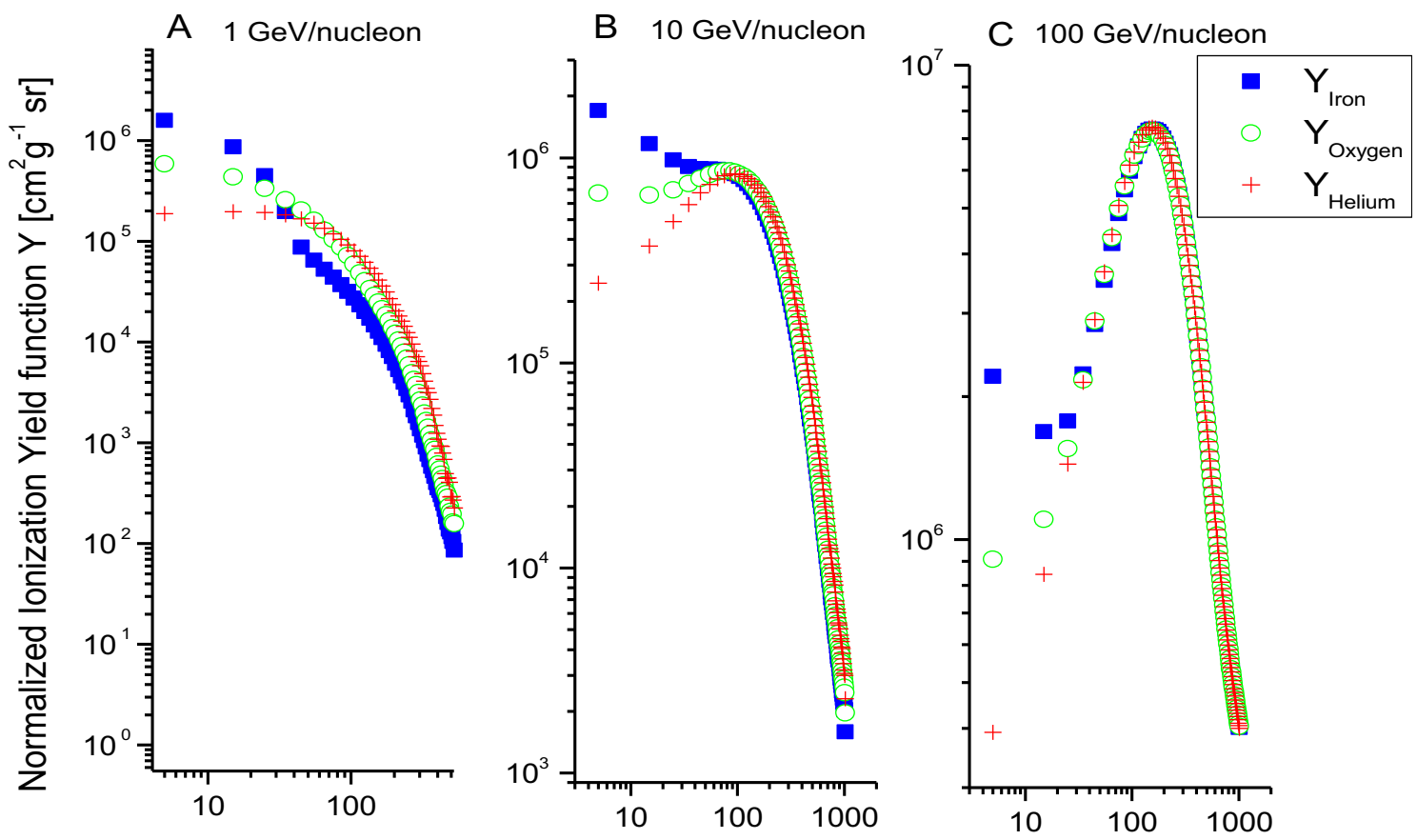

Observation depth $\left[\mathrm{g} \mathrm{cm}^{-2}\right]$

Fig. 11. Normalization of ionization yield function $Y$ for various nuclei as a function of the energy of the primary nuclei.

Earth's environment (Miroshnichenko 2008; Vainio et al. 2009). In general such events are low energy and are not able to initiate atmospheric cascades. Their ionization effect is limited to the upper polar atmosphere. Hence the studies of the effects caused by SEP are usually limited to the upper atmo- sphere above $30 \mathrm{~km}$. However, as was recently demonstrated (Mishev et al. 2010; Usoskin et al. 2011), during GLE, which are characterized by very high energy of solar particles capable to induce the atmospheric cascade, the ionization effect is important, specifically in polar atmosphere. 

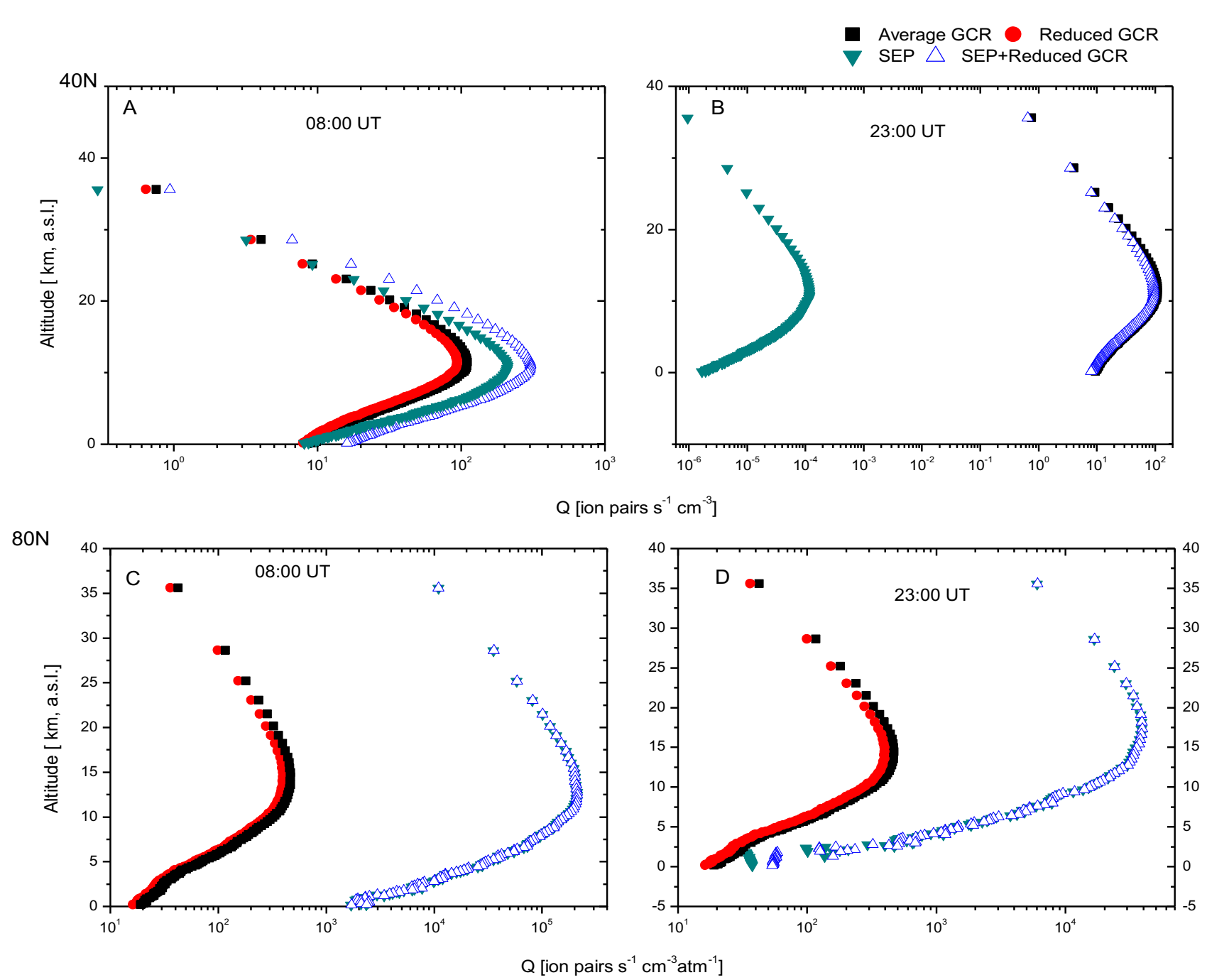

Fig. 12. Time evolution of ionization effect during GLE 69 on 20 January 2005 as function of latitude.

A well-studied GLE 69 event on 20 January 2005 was considered for analysis (Butikofer et al. 2008). The spectrum of solar protons is expressed in two different moments: at 08:00 UT a high energy part with a slope of 2.32 and at 23:00 UT low-energy part with a slope of $3.43(5,6)$. It is demonstrated (Mishev et al. 2010, 2011) that the ionization effect on event onset at 08:00 UT (Fig. 12A) is greater than that produced by delayed component at 23:00 UT (Fig. 12B). Since the event on 20 January 2005 occurred during the recovery phase of the Forbush decrease and in the following days an additional suppression of the cosmic ray intensity was observed leading to a complicated time profile of CR flux, the net ionization effect is calculated as a superposition of ion rate from solar particles and reduced galactic $\mathrm{CR}$.

In the case of $40^{\circ} \mathrm{N}$ latitude the effect at 08:00 UT is comparable to the average of galactic CR (Fig. 12A). However, the ion rates quickly decrease with altitude (below $5 \mathrm{~km}$ a.s.l.). The ionization effect due to low-energy component of the SEP spectrum, namely at 23:00 UT, is negligible (Fig. 12B). In this case the ion rates are due mostly to reduced galactic $\mathrm{CR}$ (i.e., the effect is negative after 23:00 UT). The situation is quite different for latitude $60^{\circ} \mathrm{N}$ (Mishev et al. 2011; Fig. 7). The ionization effect due to SEP at 08:00 UT is significant. The ion rates from solar particles are larger than ion rates from galactic CR by roughly an order of magnitude. The effect is significant at altitudes above about $12 \mathrm{~km}$ a.s.l. and decreases in the troposphere. The effect at 23:00 UT due to a low-energy component, as in a previous case, is negligible. Therefore the ionization effect decreases after 23:00 UT for $60^{\circ} \mathrm{N}$ latitude. In the case of $80^{\circ} \mathrm{N}$ latitude both components at 08:00 UT and at 23:00 UT cause a significant excess of ionization rates in the atmosphere. The effect at 08:00 UT is due mainly to solar protons (Fig. 12C). It is significant at altitudes above $10-12 \mathrm{~km}$ a.s.l. At 23:00 UT the effect of solar particles is significant at altitudes above $12 \mathrm{~km}$ a.s.l. and decreases in the troposphere. The ionization effect in this case is due to a reduced galactic CR (Fig. 12D). Taking into account the time evolution of the obtained ion rates, the conclusion is: the ionization effect is negative for $40^{\circ} \mathrm{N}$, especially in the troposphere and small for $60^{\circ} \mathrm{N}$, due to the accompanying Forbush decrease during the event. The ionization effect is important only in the sub-polar and polar atmosphere at high altitudes during major ground level enhancement of 20 January 2005 (Usoskin et al. 2011).

Quite recently it was demonstrated that the contribution of light nuclei, specifically Helium could be important for total ionization even at middle latitudes during GLE 69 (Mishev \& Velinov 2012). The estimated ion rate production due to Helium nuclei is comparable to the average ion rate due to galactic cosmic rays (Figs. $13 \mathrm{~A}$ and $13 \mathrm{~B}$ ) at $40^{\circ} \mathrm{N}$. It is above the ion rate production due to galactic $\mathrm{CR}$ at $60^{\circ} \mathrm{N}$ (Figs. 13C and 13D) 

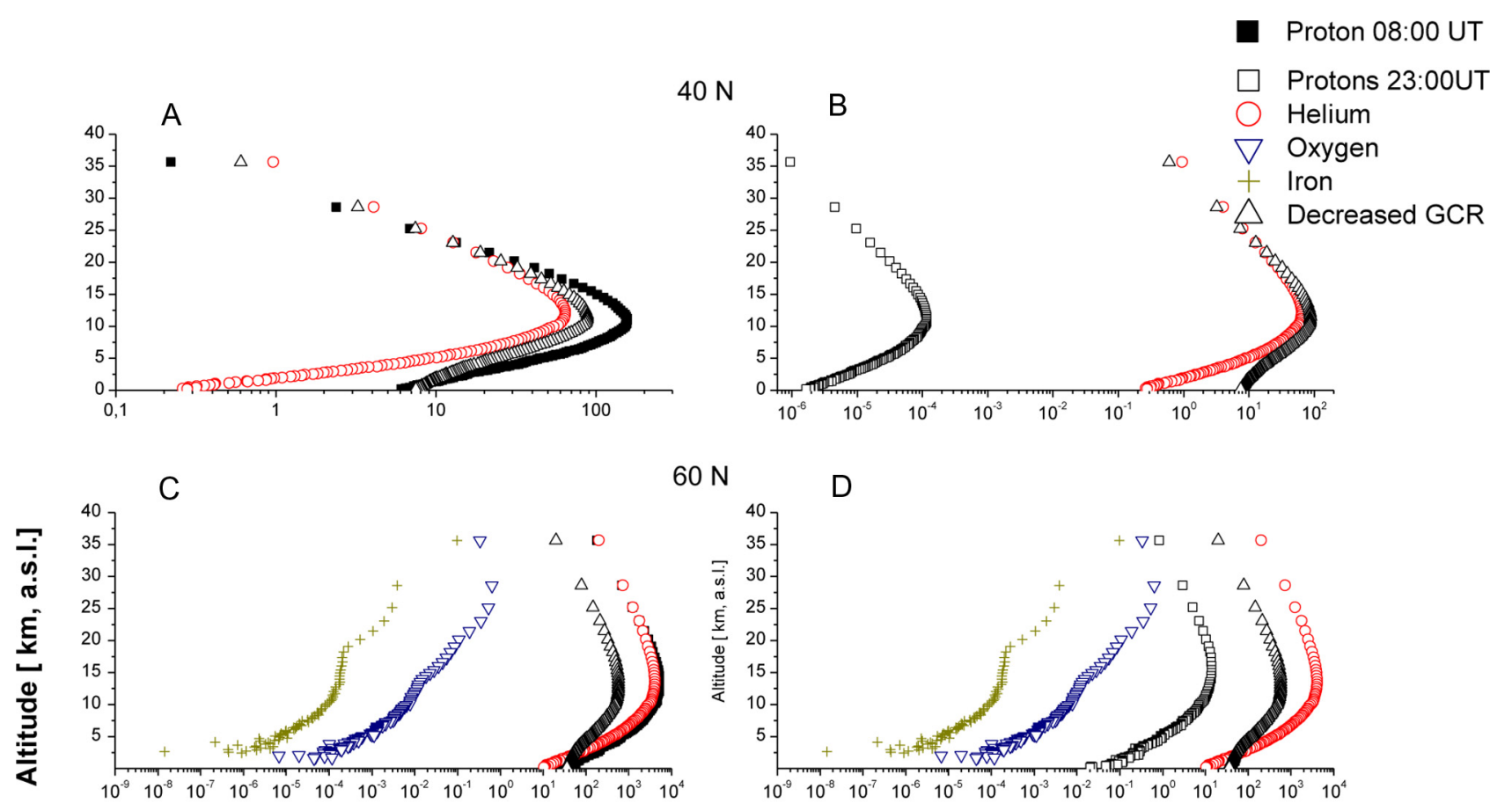

$60 \mathrm{~N}$
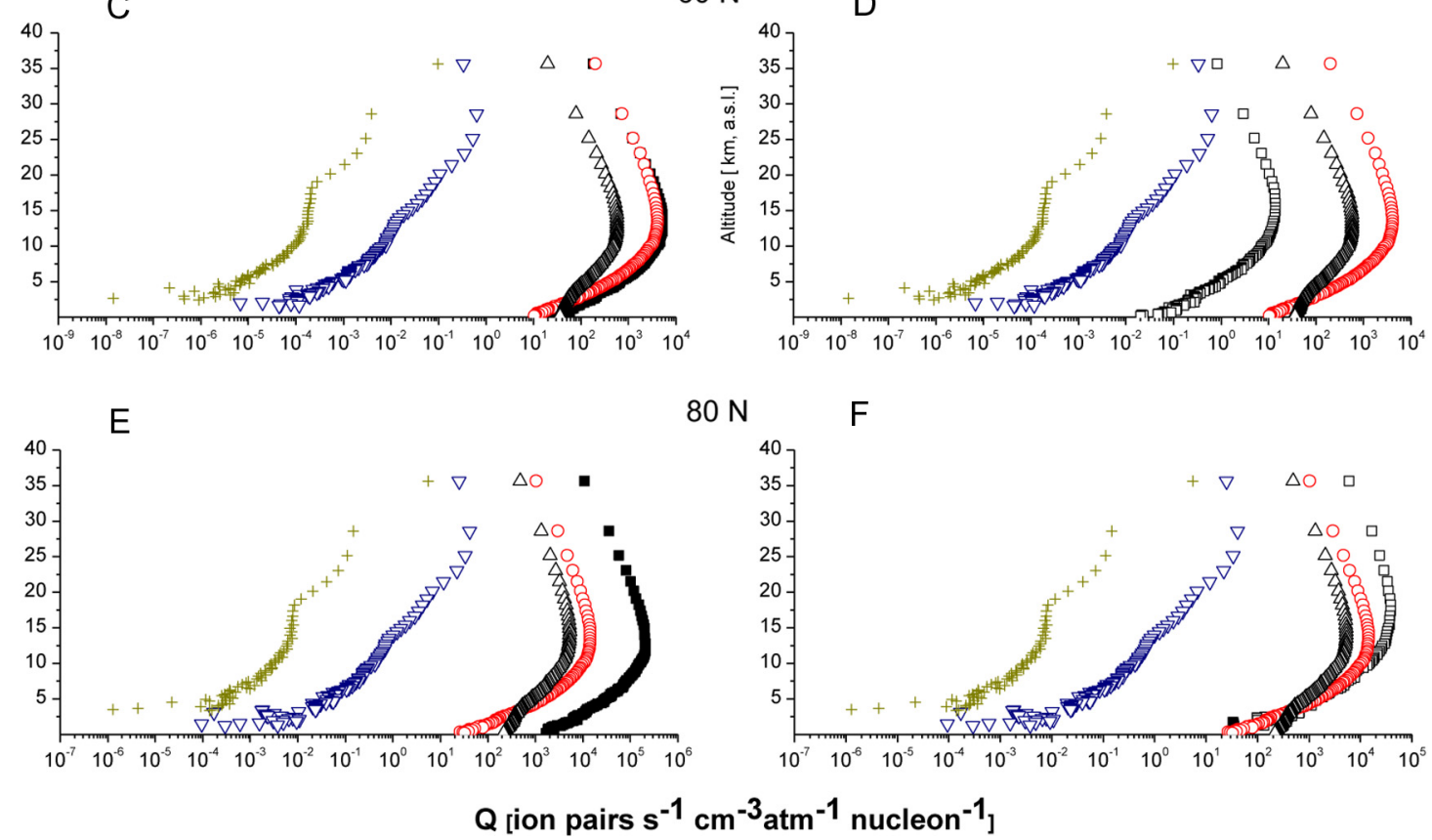

Fig. 13. Electron production rate $q(h)$ profiles on 20 January 2005 due to SEP and galactic CR for various latitudes as a function of altitude and time.

and $80^{\circ} \mathrm{N}$ (Figs. $13 \mathrm{E}$ and $13 \mathrm{~F}$ ). In addition the ion rate production due to Helium nuclei is comparable to hard proton spectrum ion rate production at $60^{\circ} \mathrm{N}$ (Fig. 13C) and soft solar proton spectrum ion rate production at $80^{\circ} \mathrm{N}$ (Fig. 13E). The ion rate due to oxygen and iron nuclei is negligible, especially in a middle and low atmosphere at $60^{\circ} \mathrm{N}$ (Figs. 13C and 13D). The medium and heavy nuclei contribute to atmospheric ionization only in the upper atmosphere at $80^{\circ} \mathrm{N}$ (Figs. 13E and 13F). In this respect the SEP event abundant on light and heavy ions deserves a special interest and further studies (Mishev et al. 2012).

\section{Applications of ionization models for estimation of CR influence on atmosphere}

Considerations of the role of $\mathrm{CR}$ and their variations in general lead to observations in last decades of different types of correlative relations between GCR and atmospheric processes responsible for climate formation. An important fact is the presence of correlation between the GCR flux and the global cloud coverage which controls the albedo (Svensmark 1998): the coverage is larger by higher GCR flux, i.e. during low solar activity. The existence of this correlation may thus express a link between solar activity and climate. However, the relation between cloudness and GCR is still a matter of debate and some recent results, particularly those related to the last deep and long solar minimum, make this relation questionable (e.g., Agee et al. 2012).

\subsection{CR influence on minor atmospheric constituents}

The influence of GCR, ACR and SEP should not be neglected in investigations of the tropospheric and stratospheric chemistry and dynamics. The CR ionization models show that the effects of these particles on the atmosphere are statistically significant in large geographic regions and for a number of relevant atmospheric species.

In a recent study (Calisto et al. 2011) based on application of 3-D Chemistry Climate Model SOCOL v2.0 (Egorova et al. 2005) and CRAC ionization model (Usoskin \& Kovaltsov 2006) statistically significant effects of GCR on tropospheric and stratospheric ozone $\mathrm{O}_{3}, \mathrm{NO} x, \mathrm{HO} x$ (on annual time scale) were found. It was estimated (Vitt \& Jackman 1996) that GCR produce $3.0-3.7 \times 10^{33}$ molecules of odd nitrogen per year in the global stratosphere, which amounts to about $10 \%$ of the $\mathrm{NO} x$ production following $\mathrm{N}_{2} \mathrm{O}$ oxidation. In addition 
the northern subpolar stratosphere is supplied with NOx in equal amounts by GCR $\left(7.1-9.6 \times 10^{32}\right.$ molecules $\left.\mathrm{yr}^{-1}\right)$ and by $\mathrm{N}_{2} \mathrm{O}$ oxidation $\left(9.4-10.7 \times 10^{32}\right.$ molecules $\left.\mathrm{yr}^{-1}\right)$.

Comparing annual mean response of the zonal mean (Calisto et al. 2011) demonstrates that the GCR-induced NOx increase to exceed $10 \%$ in the tropopause region $(20 \%$ in a polar region), whereas $\mathrm{HO} x$ decreases of about $3 \%$ caused by enhanced conversion into $\mathrm{HNO}_{3}$. As a consequence, ozone is increasing by up to $3 \%$ in the relatively unpolluted southern troposphere, where its production is sensitive to additional $\mathrm{NO} x$ from GCRs. Conversely, in the northern polar lower stratosphere, GCR are found to decrease $\mathrm{O}_{3}$ by up to $3 \%$, caused by the additional heterogeneous chlorine activation via $\mathrm{ClONO}_{2}+\mathrm{HCl}$.

The modelling of the ionization state of the atmosphere helps to study different aspects of the CR influence on the Earth atmosphere and respectively Earth's climate. The statistically determined relations between GCR and climate (on the one site) and lower stratospheric ozone (on the other) hint on the role of $\mathrm{O}_{3}$ as a mediator of GCR influence on climate Kilifarska (2012a, 2012b). This idea has been evolved further in two directions: (i) detailed inventory of the lower stratospheric ion-molecular chemistry in order to determine the energetically effective reactions; (ii) suggestion of a new mechanism translating the ozone influence down to the surface. Kilifarska (2013) suggests that the long-term variations of $\mathrm{CR}$ intensity and $\mathrm{CR}$ ionization appear to be a real driver of $\mathrm{O}_{3}$ variability in the lower stratosphere.

Another cosmic ray effect influencing on ozone are the Forbush decreases of GCR. The Forbush decreases are causing the geomagnetic storms which are a primary phenomenon among space weather phenomena. Strong geomagnetic storms are as a rule associated with Forbush decreases of galactic CR. They produce large disturbances in the ionosphere, but they affect also the neutral atmosphere including the total ozone in the middle atmosphere and troposphere (Laštovička \& Križan 2005, 2009).

Sufficiently strong, statistically significant effects of geomagnetic storms appear to occur in the total ozone at the northern higher middle latitudes only for strong events $\left(A_{\mathrm{p}}>60\right)$, in winter, and under the high solar activity and the east phase of the QBO (hereafter E-max) conditions. They occur around the $50^{\circ} \mathrm{N}$ latitudinal circle, but not around $40^{\circ} \mathrm{N}$ and $60^{\circ} \mathrm{N}$. The effect is very regional; it is strongest and statistically significant at the 3- $\sigma$ level only in the North Atlantic-European sector, where it occurs as a substantial increase of total ozone by 40-50 D.U. and more, not in other longitudinal sectors. The number of events under E-max conditions is small, but the observed effect repeats in all events without exception. The total ozone is enhanced for about 4-5 days. It should be mentioned that small changes of total ozone of the order of 510 D.U. are not reliably detectable with respect to noisy background (Laštovička \& Križan 2005, 2009). Figure 14 illustrates for Europe, $50^{\circ} \mathrm{N}$, how with more favourable conditions the effect of strong Forbush decreases/geomagnetic storms in total ozone is becoming better pronounced.

So the Forbush decreases of galactic cosmic rays seem to play a very important, likely decisive role in the effects of geomagnetic storms on total ozone. Strong geomagnetic storms occur very rarely without a corresponding Forbush decrease, we found only one such event under E-max winter conditions over 25 years but in this case a detectable effect in total ozone was absent. On the other hand, a few events were found when

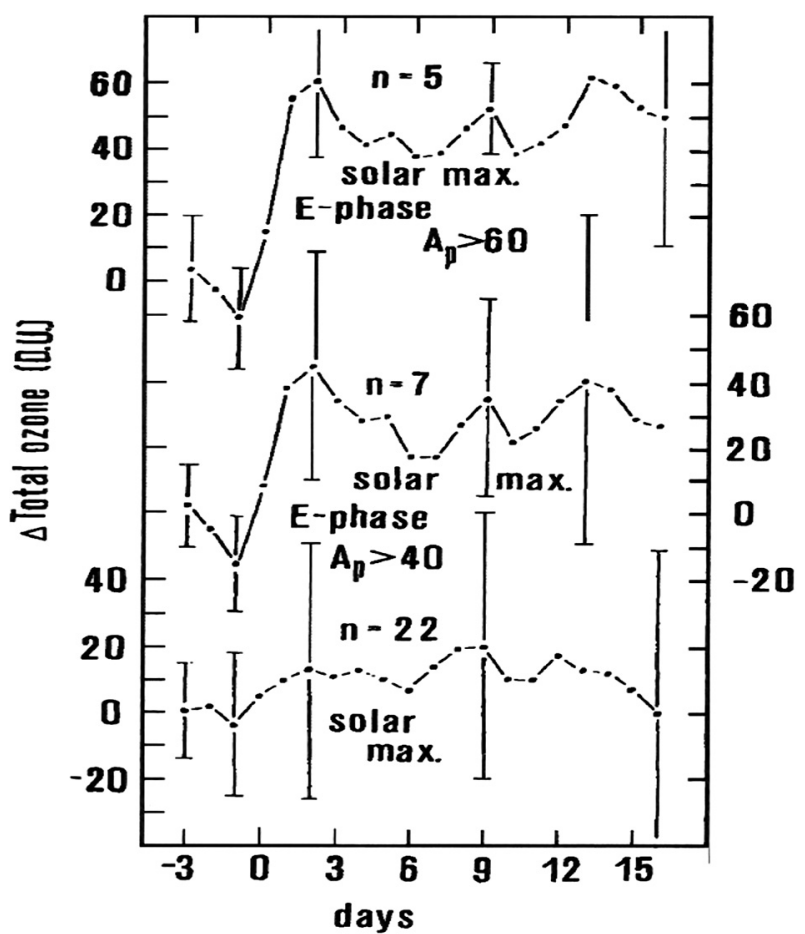

Fig. 14. Total ozone (Europe - near $50^{\circ} \mathrm{N}$ ) deviations from average level over days -3 to 15 for major geomagnetic storms under the high solar activity conditions in winter. All events under high solar activity (bottom curve), events under the E-phase of QBO (E-QBO; middle curve), very strong storms $(A \mathrm{p}>60)$ under the E-QBO (top curve). Vertical lines - error bars; $n$ - number of events.

the strong Forbush decrease occurred without a stronger geomagnetic storm, and their effects in total ozone were comparable with effects of strong geomagnetic storms accompanied by strong Forbush decreases (Laštovička \& Križan 2005, 2009).

\section{2. $C R$ influence on atmospheric electric processes}

By searching of physical mechanisms responsible for the solarterrestrial relationships, the global atmospheric electric circuit (AEC) (Bering III et al. 1998; Rycroft et al. 2000, 2007; Williams 2009) was first put forward as a candidate for a mechanism responsible for Sun-weather relationships by Markson \& Muir (1980). This suggestion received strong observational support by Tinsley \& Heelis (1993), and was developed further in a series of studies (e.g., Tinsley 2000; Harrison 2004; Tinsley 2012). Climate formation processes have been considered as depending on the rate of creation of electric charges at cloud tops by the ionosphere-ground (air-earth) current in AEC which can be sensitive to $C R$ variations. These results demonstrate the need for studying the response of AEC to the modulations of CR by solar wind.

The influence of CR on AEC is realized mainly through the atmospheric conductivity which is a result of ionization. GCR of energies $<10^{11} \mathrm{eV}$ are the only factor of ionization of the air between $5 \mathrm{~km}$ and $35 \mathrm{~km}$, and have a contribution to the ionization up to $90 \mathrm{~km}$ in the daytime and up to $100 \mathrm{~km}$ at night, i.e. GCR are necessary for creation and maintenance of AEC. Their 11-year variations during the solar cycle lead to changes in stratospheric conductivity, so that it is larger during solar minimum than during solar maximum, respectively. The relative factor of solar cycle change of the stratospheric conductivity is about $3 \%$ at equatorial, $10 \%$ at tropics, $20 \%$ at middle, 
and $50 \%$ at high and polar latitudes (according to the results of Velinov \& Mateev 1990). This leads to a small decrease of the average air-earth current at polar latitudes during solar maximums compared to solar minimums. At equatorial and low latitudes the variation of the air-earth current will be yet smaller. Larger atmospheric conductivity changes, involving larger range of altitudes (possibly, tropospheric) take place during a Forbush decreases of GCR and especially during a SEP events.

For the goal of investigations of complex relations between the solar activity, cosmic rays, and AEC, which can be a part of mechanisms of Sun-climate links, a numerical model CORIAEC (COsmic Radiation Influence on AEC) is developed (Velinov \& Tonev 2008; Tonev \& Velinov 2011; Tsagouri et al. 2013). This model serves to evaluate the electric fields and currents superimposed to AEC generated as a result of the transpolar ionospheric potential difference.

The model CORIAEC is designed to evaluate the combined influence of both the cosmic rays and the solar wind to the electric currents and fields superimposed to AEC due to the transpolar potential. CORIAEC is a 3D physically based simulation model which estimates the influence of the solar wind on the superimposed electric characteristics. These last depend on the parameters of IMF and solar wind, on one hand, and on conductivity of the ionosphere (which is sensitive to the SEP and ACR) and meso/stratosphere (where GCR has a contribution). To represent the link of the IMF and solar wind to the transpolar electric potentials and fields in CORIAEC, the model of Weimer (1996) is used which provides real-time evaluations of these characteristics in the ionosphere above the model domain. It is estimated by CORIAEC that in the polar mesosphere the superimposed electric field can have variations of up to tens of $\mathrm{mV} / \mathrm{m}$, and the air-earth current density at surface can vary by few tens of percent (Tonev \& Velinov 2011).

The observations of the air-earth current density in balloon measurements by Olson (1983) over Lake Superior in the USA (situated at high geomagnetic latitude) showed 20-40\% changes over a solar cycle. These experimentally obtained changes are somewhat larger than those estimated by the CORIAEC model. The difference between the model and the experimental results may take place possibly due to the overidealized representation of the conductivity in the model, where clean air conductivity is assumed. Actually, at polar latitudes the conductivity in the upper troposphere and lower stratosphere is dramatically reduced due to the presence of aerosols in those regions, especially after volcanic eruptions (Tinsley \& Zhou 2006). One can also note that, because of the strong reduction of the stratospheric conductivity, the contribution of its variations during a solar cycle due to the GCR flux modulation significantly increases, since the stratospheric conductivity becomes important in formation of the ionosphere-ground columnar resistance.

\subsection{CR influence on technological systems}

The investigation of impact of cosmic rays and solar energetic particles on the Earth's environment is important not only for the atmospheric processes but also for the technological and even biological systems. Ions accelerated to several tens to hundreds of $\mathrm{MeV}$ are very important for the radiation hazard effects during solar radiation storms with electronic element failures on satellites, communication and biological consequences (Kudela et al. 2000). Before their arrival a network by several stations operating in real time can provide useful alerts several minutes to tens of minutes in advance. CR and SEP of lower energy interact with the material of the satellites, spacecrafts and airplanes, and may cause the failures. There is variety of effects with consequences on the reliability of the electronic elements. The energy deposition in materials results in permanent damage in silicon semiconductor devices. This is another reason to study in detail the cosmic ray variability and the corresponding ionization effects (Kudela et al. 2010).

\section{Conclusion}

In this paper we present the results from CORIMIA and CORSICA programs and their application to calculations of the GCR, ACR and SEP ionization in ionosphere and atmosphere. Because of the different differential spectra of these types of particles, three main submodels are created. These different spectra determined different impact on the ionization in the Earth's environment.

The ionization rate profile values, which are calculated with CORIMIA, depend on the altitude, neutral density, atmospheric cut-offs, ionization losses, geomagnetic cut-offs and spectra of the particles.

CORIMIA is able to compute profiles for experimentally measured differential spectra. The evaluation of the spectra and the basic statement of the model have been discussed by Velinov et al. (2011a, 2011b, 2012). In the case with six characteristic energy intervals we reach the accuracy which is in accordance with experimental data (Brasseur \& Solomon 2005; Velinov \& Mateev 2008a, 2008b). The model can be applied for different input spectra in interactive mode and for different planetary atmospheres.

The SEP spectra at lower latitudes cause smaller values of the ionization rate profiles (Figs. 6-8 and 13) because of the higher geomagnetic cut offs. The maxima do not occur here because the atmospheric cut offs value switching appears at lower altitudes. It must be noted that the magnitude of differential spectrum dominates at higher altitudes, while at lower altitudes the number of particles with smaller exponent dominates. This is due to the greater atmospheric cut-offs which dominate over geomagnetic cut-offs for low altitudes. The powerful event GLE05 has characteristic maximum for geomagnetic latitude $\lambda_{\mathrm{m}}=75^{\circ}$.

In the region below $30 \mathrm{~km}$ the nuclear interactions of cosmic rays with air molecules must be taken into account. It is realized with the program CORSIKA (Sect. 3). Above $30 \mathrm{~km}$ we use the CORIMIA program, because the CORSIKA results are not reliable owing to the small statics. In the transition region $(25-30 \mathrm{~km})$ the CORSIKA results are in accordance with those from CORIMIA.

The simulations from CORIMIA and CORSICA are used for quantitative interpretation of $\mathrm{CR}$ impact on the ionization, chemical (ozone and other minor constituents) and electrical state of the ionosphere and atmosphere.

The application of Monte-Carlo methods for investigation of cosmic ray ionization is important, because it is possible to consider explicitly the hadron component and therefore to estimate effects in the lower $(0-10 \mathrm{~km})$ and middle $(10-100 \mathrm{~km})$ atmosphere. As was recently demonstrated, their application in specific, realistic conditions (Mishev \& Velinov 2008, 2010) permits detailed study of the ionization effect, especially at different altitudes (Mishev et al. 2010; Usoskin et al. 2011).

In addition to the well-studied significant upper atmosphere ionization effect during GLE 69 on 20 January 2005, it is 
shown that ionization effect is significant at sub-polar and polar atmosphere (Mishev et al. 2011; Usoskin et al. 2011), with fast tropospheric decrease. Moreover, it is demonstrated that the ionizaton effect at low altitudes may be negative due to Forbush decrease of GCR at middle latitudes. Therefore the effect of sporadic solar energetic particle events is limited on a global scale but most energetic events could be strong locally, particularly in sub-polar and polar region, affecting the physicalchemical properties of the upper atmosphere. Since the large SEP events leading to GLEs are different in spectra and composition, their detailed study is connected with detailed information about heliospheric and geospace conditions. In this connection extension of the existing models to the upper atmosphere is very important, as well as their comparison with analytical models.

General agreement exists that cosmic ray ionization due to $\mathrm{CR}$ and SEP influences the ozone concentration in the atmosphere. As mentioned above, the ionization effect due to SEPs is important only in a sub-polar and polar atmosphere. This is important for studies of the variations of minor atmospheric constituents. The 20 January 2005 SEP event caused large enhancements in the northern polar $\mathrm{HO}_{x}$ and $\mathrm{NO}_{x}$ constituents in the mesosphere, which lead to ozone decrease of the order of $40 \%$. However on the basis of the obtained ion rates it is demonstrated that the tropospheric and stratospheric ionization effects due to SEP are important on short to medium time scales in polar atmosphere, and they are almost negative compared to the average GCR ionization on medium time scales at middle latitudes due to the accompanying Forbush decrease. Additional more detailed studies on the influence on minor components of the atmosphere deserve special interest.

Thus, a new methodology is presented to study the CR ionization of the ionosphere and atmosphere in full detail using realistic analytical and numerical models calibrated by direct observations.

Acknowledgements. This article is the output of SG1.1 "Progress in scientific understanding of space weather" of the European COST Action ES0803 "Developing space weather products and services in Europe". The authors are grateful to COST Action ES0803 for the permanent interest and support to our SWSC studies. We thank Prof. Klaus Scherer and an anonymous referee for reviewing this paper and their comments and suggestions which helped us to improve this paper.

\section{References}

Agee, E.M., K. Kiefer, and E. Cornett, Relationship of lower troposphere cloud cover and cosmic rays: an updated perspective, J. Clim., 25 (3), 1057-1060, 2012.

Agostinelli, S., J. Allison, K. Amako, J. Apostolakis, H. Araujo, et al., GEANT 4 - a simulation toolkit, Nucl. Instrum. Methods Phys. Res., A: Accelerators, Spectrometers, Detectors and Associated Equipment, 506 (3), 250-303, 2003.

Alcaraz, J., B. Alpat, G. Ambrosi, H. Anderhub, L. Ao, et al., Cosmic protons AMS collaboration, Phys. Lett., B 490, 27, 2000a.

Alcaraz, J., B. Alpat, G. Ambrosi, H. Anderhubag, L. Ao, et al., Helium in near Earth orbit AMS collaboration, Phys. Lett., B 494, 193, 2000 b.

Battistoni, G., S. Muraro, P.R. Sala, F. Cerutti, A. Ferrari, et al., M., Albrow, and R. Raja, The FLUKA code: description and benchmarking. in Proc. of the Hadronic Shower Simulation Workshop 2006, Fermilab 6-8 September 2006, 896: AIP Conference Proc, 31-49, 2007.

Bazilevskaya, G.A., I.G. Usoskin, E.O. Fluckiger, R.G. Harrison, L. Desorgher, et al., Cosmic ray induced ion production in the atmosphere, Space Sci. Rev., 137, 149-173, 2008.
Bering III, E.A., A.A. Few, and J.R. Benbrook, The global electric circuit, Phys. Today, 51 (10), 24, 1998.

Boezio, M., P. Carlson, T. Francke, N. Weber, M. Suffert, et al., The cosmic ray proton and helium spectra between 0.4 and $200 \mathrm{GV}$, Astrophys. J., 518, 457, 1999.

Brasseur, G., and S. Solomon, Aeronomy of the Middle Atmosphere, Springer, Dordrecht, 2005.

Buchvarova, M., and P.I.Y. Velinov, Modeling spectra of cosmic rays influencing on the ionospheres of earth and outer planets during solar maximum and minimum, J. Adv. Space Res., 36 (11), 2127 2133, 2005.

Buchvarova, M., and P.I.Y. Velinov, Empirical model of cosmic ray spectrum in energy interval $1 \mathrm{MeV}-100 \mathrm{GeV}$ during 11 -year solar cycle, J. Adv. Space Res., 45 (8, 1), 1026-1034, 2010.

Buchvarova, M., P.I.Y. Velinov, and I. Buchvarov, Model approximation of cosmic ray spectrum, Planet. Space Sci., 59 (4), 355 363, 2011.

Burger, R.A., M.S. Potgieter, and B. Heber, Rigidity dependence of cosmic ray proton latitudinal gradients measured by the Ulysses spacecraft: implications for the diffusion tensor, J. Geophys. Res., 105, 27447, 2000.

Butikofer, R., E.O. Fluckiger, L. Desorgher and M.R. Moser, The extreme solar cosmic ray particle event on 20 January 2005 and its influence on the radiation dose rate at aircraft altitude, Sci. Total Environ., 391 (2-3), 177-183, 2008.

Calisto, M., I. Usoskin, E. Rozanov, and T. Peter, Influence of galactic cosmic rays on atmospheric composition and dynamics, Atmos. Chem. Phys., 11, 4547-4556, 2011.

Cummings, A.C., E.C. Stone, and W.R. Webber, Evidence that the anomalous cosmic-ray component is singly ionized, Astrophys. $J$., 287, 99-103, 1984.

Desorgher, L., E. Fluckiger, M. Gurtner, M.R. Moser, R. Bütikofer, et al., Atmocosmics: a GEANT4 code for computing the interaction of cosmic rays with the Earths atmosphere, Int. J. Mod. Phys., A 20 (29), 6802-6804, 2005.

Dorman, L.I., Cosmic Rays in the Earth's Atmosphere and Underground, Kluwer Academic Publishers, Dordrecht, 2004.

Dorman, L.I., and I.D. Kozin, Cosmic Radiation in the Upper Atmosphere, Fizmatgiz, Moscow, 1983.

Dorman, L.I., and T.M. Krupitskaya, Calculation of expected ratio of solar cosmic ray ion generation speeds on different altitudes, in Cosmic Rays, Nauka, Moscow, 15, 30-33, 1975.

Egorova, T., E. Rozanov, V. Zubov, E. Manzini, W. Schmutz, and T. Peter, Chemistry-climate model SOCOL: a validation of the present-day climatology, Atmos. Chem. Phys., 5, 1557-1576, DOI: 10.5194/acp-8-6365-2005, 2005.

Ferrari, A., and P. Sala, ATLAS Int. Note PHYS-No-086, CERN, Geneva, 1996.

Fesefeldt, H.C., GHEISHA program, Technical Report PITHA 8502, III Physikalisches Institut, RWTH Aachen Physikzentrum, 5100 Aachen, Germany, September, 1985.

Ginzburg, V.L., and S.I. Syrovatskii, The Origin of the Cosmic Rays, Pergamon Press, Oxford, 1964.

Harrison, R.G., The global atmospheric electrical circuit and climate, Sur. Geophys., 25 (5-6), 441-484, 2004.

Heck, D., J. Knapp, J.N. Capdevielle, G. Schatz and T. Thouw, CORSIKA: A Monte Carlo Code to Simulate Extensive Air Showers, Forschungszentrum Karlsruhe Report FZKA 6019, 1998.

Hillas, A.M., Cosmic Rays, Pergamon Press, Oxford, 1972.

Keilhauer, B., J. Blumer, R. Engel, H.O. Klages and M. Risse, Impact of varying atmospheric profiles on extensive air shower observation: atmospheric density and primary mass reconstruction, Astropart. Phys., 22 (3-4), 249-261, 2004.

Keilhauer, B., J. Blumer, R. Engel and H.O. Klages, Impact of varying atmospheric profiles on extensive air shower observation: fluorescence light emission and energy reconstruction, Astropart. Phys., 25 (4), 259-268, 2006.

Kilifarska, N.A., Climate sensitivity to the lower stratospheric ozone variations, J. Atmos. Sol. Terr. Phys., 90/91, 9-14, 2012a. 
Kilifarska, N.A., Ozone as a mediator of galactic cosmic ray influence on climate, Sun Geosphys., 7 (2), 97-102, 2012b.

Kilifarska, N.A., An autocatalytic cycle for ozone production in the lower stratosphere initiated by Galactic Cosmic rays, C.R. Acad. Bulg. Sci., 66 (2), 243-252, 2013.

Krivolutsky, A., A. Kuminov, and T. Vyushkova, Ionization of the atmosphere caused by solar protons and its influence on ozonosphere of the Earth during 1994-2003, J. Atmos. Sol. Terr. Phys., 67, 105-117, 2005.

Kudela, K., On energetic particles in space, Acta Phys. Slovaca, 59, 537-652, 2009.

Kudela, K., M. Storini, M.Y. Hofer, and A. Belov, Cosmic rays in relation to space weather, Space Sci. Rev., 93 (1-2), 153-174, 2000.

Kudela, K., H. Mavromichalaki, A. Papaioannou, and M. Gerontidou, On mid-term periodicities in cosmic rays, Sol. Phys., 266, 173-180, 2010.

Laštovička, J., and P. Križan, Geomagnetic storms, Forbush decreases of cosmic rays and total ozone at northern higher middle latitudes, J. Atmos. Sol. Terr. Phys., 67, 119-124, 2005.

Laštovička, J., and P. Križan, Impact of strong geomagnetic storms on total ozone at southern higher middle latitudes, Stud. Geophys. Geod., 53, 151-156, 2009.

Leske, R.A., A.C. Cummings, R.A. Mewaldt, and E.C. Stone, Anomalous and galactic cosmic rays at $1 \mathrm{AU}$ during the cycle 23/24 solar minimum, Space Sci. Rev., DOI: 10.1007/s11214-011-9772-1, 2011.

Markson, R., and M. Muir, Solar wind control of the Earth's electric field, Science, 208, 979-990, 1980.

McDonald, F.B., B. Klecker, R.E. McGuire, and D.V. Reames, Relative recovery of galactic and anomalous cosmic rays at $1 \mathrm{AU}$ : further evidence for modulation in the heliosheath, J. Geophys. Res., 107 (A8), DOI: 10.1029/2001JA000206, 2002.

Menn, W., M. Hof, O. Reimer, M. Simon, A.J. Davis, et al., The absolute flux of protons and helium at the top of the atmosphere using IMAX, Astrophys J., 533, 281, 2000.

Mertens, C.J., B.T. Kress, M. Wiltberger, W.K. Tobiska, B. Grajewski, X. Xu, in Atmospheric Ionizing Radiation from Galactic and Solar Cosmic Rays, Current Topics in Ionizing Radiation Research, edited by M. Dr. Nenoi, InTech, Available from: http: //www.intechopen.com/books/current-topics-in-ionizing-radiation-research/atmospheric-ionizing-radiationfrom-galactic-and-solar-cosmic-rays, 2012.

Miroshnichenko, L.I., Solar Cosmic Rays, ASSL, 260, Kluwer Academic Publishers, Dordrecht, The Netherlands, 2001.

Miroshnichenko, L.I., Solar cosmic rays in the system of solarterrestrial relations, J. Atmos. Sol. Terr. Phys., 70, 450-466, 2008.

Mishev, A., A study of atmospheric processes based on neutron monitor data and Cherenkov counter measurements at high mountain altitude, J. Atmos. Sol. Terr. Phys., 72 (16), 1195-1199, 2010.

Mishev, A., and P.I.Y. Velinov, Atmosphere ionization due to cosmic ray protons estimated with CORSIKA code simulations, C.R. Acad. Bulg. Sci., 60 (3), 225-230, 2007.

Mishev, A., and P.I.Y. Velinov, Effects of atmospheric profile variations on yield ionization function $\mathrm{Y}$ in the atmosphere, C.R. Acad. Bulg. Sci., 61 (5), 639-644, 2008

Mishev, A., and P.I.Y. Velinov, Normalized atmospheric ionization yield functions $\mathrm{Y}$ for different cosmic ray nuclei obtained with recent CORSIKA code simulations, C.R. Acad. Bulg. Sci., 62 (5), 631-640, 2009.

Mishev, A., and P.I.Y. Velinov, The effect of model assumptions on computations of cosmic ray induced ionization in the atmosphere, J. Atmos. Sol. Terr. Phys., 72, 476-481, 2010.

Mishev, A., and P.I.Y. Velinov, Renormalized ionization yield function $\mathrm{Y}$ for different nuclei obtained with full Monte Carlo simulations, C.R. Acad. Bulg. Sci., 64 (7), 997-1006, 2011 a.
Mishev, A., and P.I.Y. Velinov, Normalized ionization yield function for various nuclei obtained with full Monte Carlo simulations, $J$. Adv. Space Res., 48, 19-24, 2011b.

Mishev, A., and P.I.Y. Velinov, Contribution of cosmic ray nuclei of solar and galactic origin to atmospheric ionization during SEP event on 20 January 2005, C.R. Acad. Bulg. Sci., 65 (3), 373-380, 2012.

Mishev, A., P.I.Y. Velinov, and L. Mateev, Atmospheric ionization due to solar cosmic rays from 20 January 2005 calculated with Monte Carlo simulations, C.R. Acad. Bulg. Sci., 63 (11), 16351642,2010

Mishev, A., P.I.Y. Velinov, L. Mateev, and Y. Tassev, Ionization effect of solar protons in the Earth atmosphere - case study of the 20 January 2005 SEP event, J. Adv. Space Res., 48, 1232-1237, 2011.

Mishev, A., P.I.Y. Velinov, L. Mateev, and Y. Tassev, Ionization effect of nuclei with solar and galactic origin in the earth atmosphere during GLE 69 on 20 January 2005, J. Atmos. Sol. Terr. Phys., 89, 1-7, 2012.

Nestorov, G., Physics of the Lower Ionosphere, Publ. House of the Bulg. Acad. Sci, Sofia, 1969.

O'Brien, K., Cosmic-ray propagation in the atmosphere, Il Nuovo Cimento A, 3 (4), 521-547, 1971.

Olson, D.E., Interpretation of the solar influence on the atmospheric electrical parameters, in Weather and Climate Responses to Solar Variations, edited by B.M., McCormac, Assoc. Univ. Press, Boulder, CO, 483-488, 1983.

Porter, H.S., C.H. Jackman, and A.E.S. Green, Efficiencies for production of atomic nitrogen and oxygen by relativistic proton impact in air, J. Chem. Phys., 65, 154-167, 1976.

Press, W.H., B.P. Flannery, S.A. Teukolsky, and W.T. Vetterling, Numerical Recipes in C ++- the Art of Scientific Computing, Cambridge University Press, Cambridge, 1991.

Reid, G.S., A study of enhanced ionisation produced by solar protons during a polar cap absorption event, J. Geophys. Res., 66, 4071, 1961.

Rycroft, M.J., S. Israelson, and C. Price, The global atmospheric electrical circuit, solar activity, and climate change, J. Atmos. Sol. Terr. Phys., 62 (17-18), 1563-1576, 2000.

Rycroft, M.J., A. Odzimek, N.F. Arnold, M. Fullekrug, A. Kulak, and T. Neubert, New model simulations of the global atmospheric electrical circuit driven by thunderstorms and electrified shower clouds: the roles of lightning and sprites, J. Atmos. Sol. Terr. Phys., 69, 2485-2509, 2007.

Scherer, K., H. Fichtner, T. Borrmann, J. Beer, L. Desorgher, E. Flükiger, and H.-J. Fahr, Interstellar-terrestrial relations: variable cosmic environments, the dynamic heliosphere, and their imprints on terrestrial archives and climate, Space Sci. Rev., 127, 327-465, 2007.

Seo, E.S., J.F. Ormes, R.E. Streitmatter, S.J. Stochaj, W.V. Jones, et al., Measurement of cosmic-ray proton and helium spectra during the 1987 solar minimum, Astrophys. J., 371, 763, 1991.

Shikaze, Y., S. Haino, K. Abe, H. Fuke, T. Hams, et al., Measurements of $0.2-20 \mathrm{GeV} / \mathrm{n}$ cosmic-ray proton and helium spectra from 1997 through 2002 with the BESS spectrometer, Astropart. Phys., 28, 154, 2007.

Simpson, J.A., Cosmic radiation: particle astrophysics in the heliosphere, in Frontiers in Cosmic Physics, edited by R.B., Mendell, and A.I. Mincer, Ann. N. York Acad. Sci., 655, 95, 1992.

Singh, A.K., D. Siingh, and R.P. Singh, Space weather: physics, effects and predictability, Surv. Geophys., 31, 581-638, 2010.

Singh, A.K., D. Singh, and R.P. Singh, Impact of galactic cosmic rays on Earth's atmosphere and human health, Atmos. Environ., 45, 3806-3818, 2011.

Sternheimer, R., in Fundamental Principles and Methods of Particle Detection. Methods of Experimental Physics, vol. V. A. Nuclear Physics, edited by L.C.L., Yuan, and C.S. Wu, New York, London, Academic Press, 1961. 
Svensmark, H., Influence of cosmic rays on Earth's climate, Phys. Rev. Lett., 81 (22), 5027-5030, 1998.

Tinsley, B.A., Influence of solar wind on the global electric circuit, and inferred effects on cloud microphysics, temperature, and dynamics in the troposphere, Space Sci. Rev., 94 (1-2), 231-258, 2000.

Tinsley, B.A., A working hypothesis for connections between electrically-induced changes in cloud microphysics and storm vorticity, with possible effects on circulation, Adv. Space Res., 50, 791-805, 2012.

Tinsley, B.A., and R.A. Heelis, Correlations of atmospheric dynamics with solar activity: evidence for a connection via the solar wind, atmospheric electricity, and cloud microphysics, $J$. Geophys. Res., 98, 10375-10384, 1993.

Tinsley, B.A., and L. Zhou, Initial results of a global circuit model with stratospheric and tropospheric aerosols, J. Geophys.Res., 111, D16205, 2006.

Tonev, P.T., and P.I.Y. Velinov, Model study of the influence of solar wind parameters on electric currents and fields in middle atmosphere at high latitudes, C.R. Acad. Bulg. Sci., 64 (12), 1733-1742, 2011

Tsagouri, I., A. Belehaki, N. Bergeot, C. Cid, V. Delouille, et al., Progress in space weather modeling in an operational environment, J. Space Weather Space Clim., 3, in press., 2013

Usoskin, I.G., O.G. Gladysheva, and G.A. Kovaltsov, Cosmic ray induced ionization in the atmosphere: spatial and temporal changes, J. Atmos. Sol. Terr. Phys., 66, 1791-1796, 2004.

Usoskin, I., K. Alanko-Huotari, G. Kovaltsov, and K. Mursula, Heliospheric modulation of cosmic rays: Monthly Reconstruction for 1951-2004, J. Geophys. Res., 110 (A12), CiteID: A12108, 2005.

Usoskin, I., L. Desorgher, P.I.Y. Velinov, M. Storini, E. Flueckiger, R. Buetikofer, and G.A. Kovalstov, in Solar and Galactic Cosmic Rays in the Earth's Atmosphere. Developing the Scientific Basis for Monitoring, Modeling and Predicting Space Weather, edited by Lilensten, J., COST 724 Final Report, COST Office, Brussels, $127-135,2008$.

Usoskin, I, L. Desorgher, P.I.Y. Velinov, M. Storini, E. Flueckiger, R. Buetikofer, and G.A. Kovalstov, Solar and galactic cosmic rays in the Earth's atmosphere, Acta Geophys., 57, (1/March), 88-101, 2009.

Usoskin, I., and G. Kovaltsov, Cosmic ray induced ionization in the atmosphere: full modeling and practical applications, J. Geophys. Res., 111, D21206, 2006.

Usoskin, I.G., G.A. Kovaltsov, and I.A. Mironova, Cosmic ray induced ionization model CRAC: CRII: an extension to the upper atmosphere, J. Geophys. Res., 115, D10302, 2010.

Usoskin, I.G., G.A. Kovaltsov, I.A. Mironova, A.J. Tylka and W.F. Dietrich, Ionization effect of solar particle GLE events in low and middle atmosphere, Atmos. Chem. Phys., 11, 1979-1988, 2011.

Vainio, R., L. Desorgher, D. Heynderickx, M. Storini, E. Flückiger, et al., Dynamics of the Earth's particle radiation environment, Space Sci. Rev., 147, 187-231, 2009.

Van Allen, J.A., Physics and Medicine of the Upper Atmosphere, Chapter 14, Albuquerque: Univ. N. Mexico Press, 1952.

Velinov, P.I.Y., An expression for ionospheric electron production rate by cosmic rays, C.R. Acad. Bulg. Sci., 19 (2), 109-112, 1966.

Velinov, P.I.Y., Some results of the rate of electron production in the cosmic layer of low ionosphere, C.R. Acad. Bulg. Sci., 20 (11), 1141-1144, 1967a.

Velinov, P.I.Y., On electron production rates in the polar cap ionosphere due to solar cosmic rays, C.R. Acad. Bulg. Sci., 20 (12), 1278-1278, 1967b.

Velinov, P.I.Y., On ionization in the ionospheric $\mathrm{D}$ region by galactic and solar cosmic rays, J. Atmos. Terr. Phys., 30, 1891-1905, 1968.

Velinov, P.I.Y., Solar cosmic ray ionization in the low ionosphere, $J$. Atmos. Terr. Phys., 32, 139-147, 1970.

Velinov, P.I.Y., Cosmic ray ionization rates in the planetary atmospheres, J. Atmos. Terr. Phys., 36, 359-362, 1974.
Velinov, P.I.Y., Effect of the Anomalous Cosmic Ray (ACR) component on the high-latitude ionosphere, C.R. Acad. Bulg. Sci., 44 (2), 33-36, 1991.

Velinov, P.I.Y., and L. Mateev, Response of the middle atmosphere on galactic cosmic ray influence, Geomagn. Aeronomy, 30 (4), 593-598, 1990.

Velinov, P.I.Y., and L. Mateev, Improved cosmic ray ionization model for the system ionosphere - atmosphere. Calculation of electron production rate profiles, J. Atmos. Sol. Terr. Phys., 70, 574-582, 2008a.

Velinov, P.I.Y., and L. Mateev, Analytical approach to cosmic ray ionization by nuclei with charge $\mathrm{Z}$ in the middle atmosphere distribution of galactic CR effects, J. Adv. Space Res., 42, 15861592, 2008b.

Velinov, P.I.Y., and A. Mishev, Cosmic ray induced ionization in the atmosphere estimated with CORSIKA code simulations, C.R. Acad. Bulg. Sci., 60 (5), 495-502, 2007.

Velinov, P.I.Y., and A. Mishev, Cosmic ray induced ionization in the upper, middle and lower atmosphere simulated with CORSIKA code, in Proceedings of the 30th International Cosmic Ray Conference, Merida, Mexico, 3-11 July 2007, edited by R., Caballero, et al., Universidad Nacional Autónoma de México, Mexico City, Mexico, 1 (SH), 749-752, 2008a.

Velinov, P.I.Y., and A. Mishev, Solar cosmic ray induced ionization in the Earth's atmosphere obtained with CORSIKA code simulations, C.R. Acad. Bulg. Sci., 61 (7), 927-932, 2008 b.

Velinov, P.I.Y., and P. Tonev, Electric currents from thunderstorms to the ionosphere during a solar cycle: quasi-static modeling of the coupling mechanism, J. Adv. Space Res., 42, 569-1575, 2008.

Velinov, P.I.Y., G. Nestorov, and L. Dorman, Cosmic Ray Influence on the Ionosphere and on the Radio-Wave Propagation, BAS Publ. House, Sofia, 1974.

Velinov, P.I.Y., M. Buchvarova, L. Mateev, and H. Ruder, Determination of electron production rates caused by cosmic ray particles in ionospheres of terrestrial planets, J. Adv. Space Res., 27 (11), 1901-1908, 2001.

Velinov, P.I.Y., H. Ruder, L. Mateev, M. Buchvarova, and V. Kostov, Method for calculation of ionization profiles caused by cosmic rays in giant planet ionospheres from Jovian group, $J$. Adv. Space Res., 33, 232-239, 2004.

Velinov, P.I.Y., L. Mateev, and N. Kilifarska, 3D model for cosmic ray planetary ionization in the middle atmosphere, Annal. Geophys., 23 (9), 3043-3046, 2005a.

Velinov, P.I.Y., H. Ruder, and L. Mateev, Analytical model for cosmic ray ionization by nuclei with charge $Z$ in the lower ionosphere and middle atmosphere, C.R. Acad. Bulg. Sci., 58, 897-902, 2005b.

Velinov, P.I.Y., H. Ruder, and L. Mateev, Energy interval coupling in improved cosmic ray ionization model with three intervals in ionization losses function for the system atmosphere/ionosphere, C.R. Acad. Bulg. Sci., 59, 847-854, 2006.

Velinov, P.I.Y., L. Mateev, and H. Ruder, Generalized model of ionization profiles due to cosmic ray particles with charge $Z$ in planetary ionospheres and atmospheres with 5 energy interval approximation of the ionization losses function, C.R. Acad. Bulg. Sci., 61 (1), 133-146, 2008.

Velinov, P.I.Y., A. Mishev, and L. Mateev, Model for induced ionization by galactic cosmic rays in the Earth atmosphere and ionosphere, J. Adv. Space Res., 44, 1002-1007, 2009.

Velinov, P.I.Y., S. Asenovski, and L. Mateev, Simulation of cosmic ray ionization profiles in the middle atmosphere and lower ionosphere on account of characteristic energy intervals, C.R. Acad. Bulg. Sci., 64 (9), 1303-1310, 2011a.

Velinov, P.I.Y., A. Mishev, S. Asenovski, and L. Mateev, New operational models for cosmic ray ionization in space physics, Bulg. J. Phys., 38, 264-273, 2011 b.

Velinov, P.I.Y., S. Asenovski, and L. Mateev, Improved cosmic ray ionization model for ionosphere and atmosphere (CORIMIA) with account of 6 characteristic intervals, C.R. Acad. Bulg. Sci., 65 (8), 1135-1144, 2012. 
P.I.Y. Velinov et al.: Impact of CR and SEP on the Earth's ionosphere and atmosphere

Vitt, F.M., and C.H. Jackman, A comparison of sources of odd nitrogen production from 1974 through 1993 in the Earth's middle atmosphere as calculated using a two-dimensional model, $J$. Geophys. Res., 101, 6729-6740, 1996.

Weimer, D.R., A flexible, IMF dependent model of high latitude electric potential having "space weather" applications, Geophys. Res. Lett., 23, 2549-2552, 1996.
Williams, E.R., The global electrical circuit: a review, Atmos. Res., 91, 140-152, 2009.

Wissing, J.M., and M.B. Kallenrode, Atmospheric Ionization Module Osnabruck (AIMOS): a 3D model to determine atmospheric ionization by energetic charged particles from different populations, J. Geophys. Res., 114, A06104, 2009.

Wolfram Research Inc., Mathematica, Version 7.0, Champaign, IL, 2008 .

Cite this article as: Velinov $\mathrm{P}$, Asenovski S, Kudela K, Lastovicka J, Mateev L, et al.: Impact of cosmic rays and solar energetic particles on the Earth's ionosphere and atmosphere. J. Space Weather Space Clim., 2013, 3, A14. 